Nuclear Physics A445 (1985) 263-303

North-Holland Publishing Company

\title{
EXTENDED THOMAS-FERMI THEORY AT FINITE TEMPERATURE
}

\author{
J. BARTEL and M. BRACK \\ Institute of Theoretical Physics, University of Regensburg, D-8400 Regensburg, FRG \\ M. DURAND \\ Institut des Sciences Nucléaires*, F-38026 Grenoble, France \\ Received 18 March 1985 \\ (Revised 14 June 1985)
}

\begin{abstract}
We present a generalization of the extended Thomas-Fermi (ETF) theory to finite temperatures $T$. Starting from the Wigner-Kirkwood expansion of the Bloch density in powers of $\hbar$, we derive the gradient expansion of the free energy and entropy density functionals $\mathscr{F}[\rho]$ and $\sigma[\rho]$ up to fourth order with thcir correct temperaturc-dependent cocfficients. (Effective mass and spin-orbit contributions are taken into account up to second order.) For a harmonic-oscillator potential we show that both the $\hbar$-expansion of the free energy and the entropy and the gradient expansion of the functionals $\mathscr{F}[\rho]$ and $\sigma[\rho]$ converge very fast and yield the exact quantum-mechanical results for $k T \gtrsim 3 \mathrm{MeV}$, where the shell effects are washed out. Finally we discuss the Euler vanational equation obtained with the new functionals and use its numerical solutions for semi-infinite symmetric nuclear matter to test the quality of parametrized trial densities. As an application. we present liquid-drop model parameters, calculated with a realistic Skyrme interaction, as functions of the temperature.
\end{abstract}

\section{Introduction}

A lot of interest has recently been focussed on highly excited nuclear systems in which the excitation energy is equilibrized amongst the nucleons. In that case a thermodynamical-statistical description is adequate and the excitation can be expressed in terms of an intrinsic temperature. Such "hot nuclei" can nowadays be produced as compound systems in energetic heavy-ion collisions, and this provides a challenge to look for observable temperature dependences of their properties. One interesting speculation is to observe possible consequences of a phase-transition between liquid and gaseous nuclear matter, such as e.g. the associated critical tcmpcraturc ${ }^{1}$ ). Another possibility is to measure the expected increase of the fissility of nuclei with increasing temperature ${ }^{2}$ ).

In astrophysics, the equation of state of hot nuclear matter can play a crucial role for the formation of supernovae during the gravitational collapse of massive stars,

\footnotetext{
* Laboratoire associé au CNRS.
} 
where condensed nuclei can coexist with a nucleon gas in local thermodynamical equilibrium ${ }^{3}$ ).

The theoretical description of such hot systems with condensed (liquid) and gaseous components is conceptually easier in the case of stars with their practically infinite extension where it is possible to spcak of phase transitions under (local) equilibrium conditions. In isolated hot compound nuclei such as are created in a heavy-ion collision, phase transitions do not strictly exist due to their finiteness. Their theoretical treatment is furthermore complicated by their instability due to nucleon evaporation, i.e. by the lack of an equilibrium situation. However, the relatively long evaporation times at not too high excitations may justify treating these systems like classical, superheated (and thus, metastable) liquid drops ${ }^{4}$ ). In this sense, variational calculations within a static mean-field or Hartree-Fock (HF) theory, together with the use of suitable boundary conditions, might be a sufficiently well-justified procedure for the description of hot, metastable nuclei.

HF calculations for finite nuclei at finite temperature $T$ were done for the first time about a decade ago ${ }^{5,6}$ ). At that time they were of a rather academic interest and the occupation of the continuum states, which leads to evaporation, was only given an approximate treatment. What was clearly demonstrated there in a selfconsistent way is the fact that the shell effects cease to exist - due to the smearing out of the Fermi surface - at a typical temperature $T \approx 2.5-3 \mathrm{MeV}$ which is roughly independent of the size of the nucleus. More recently, such calculations were taken up again for astrophysical applications, whereby periodic boundary conditions in the Wigner-Seitz approximation were used ${ }^{7,8}$ ). The same technique was also used to describe an isolated, heated nucleus ${ }^{9}$ ); hereby the necessity was pointed out for the use of some subtraction procedure in order to obtain results which are independent of the size of the boundary at $T \geq 4 \mathrm{MeV}$ where the occupation of continuum states becomes important.

Such HF calculations with the correct treatment of the continuum states become extremely time-consuming at higher temperatures, in particular if non-spherical boundary conditions are imposed in connection with the Wigner-Seitz approximation ${ }^{8}$ ). On the other hand, there is necessarily a fair amount of redundancy in the microscopic description of such a system for $T \geq 3 \mathrm{MeV}$ where the shell effects are washed out and all expectation values become smooth functions of particle numbers and deformation. In this case a semiclassical treatment in terms of densities and average fields is not only sufficient, but also much more economical and physically transparent. The development and discussion of such a semiclassical method for $T>0$ is the purpose of the present paper.

Semiclassical variational calculations for average ground-state properties and deformation energies of nuclei at $T=0$ have recently become very successful, particularly in connection with Skyrme-type effective interactions. [For an extended review of such calculations, see ref. ${ }^{10}$ ) and the literature quoted therein.] The perturbative inclusion of shell effects by the Strutinsky method ${ }^{11}$ ) has been justified 
numerically within the HF framework ${ }^{12}$ ). The technically most refined of these semiclassical methods is the density variational method using local density functionals for the kinetic energy and spin densities in a gradient expansion, derived from the so-called extended Thomas-Fermi (ETF) model $^{13}$ ), and is described in detail in ref. ${ }^{10}$ ). The density variational method itself is practically as old as nuclear physics ${ }^{14}$ ); its formal justification was, however, only given in 1964 by the Hohenberg-Kohn theorem ${ }^{15}$ ). The importance of fourth-order gradient corrections in the kinetic energy density functional $\tau[\rho]$ was pointed out only recently ${ }^{16}$ ) and made particularly evident in semiclassical calculations of fission barriers ${ }^{10,17}$ ).

The extension of the Hohenberg-Kohn theorem to fermion systems at finite temperature ${ }^{18}$ ) encourages one to seek local density functionals for energy and entropy which are valid at $T>0$. Whereas the Thomas-Fermi theory at $T>0$ has been known for a long time ${ }^{19}$ ) and has been used for infinite ${ }^{20}$ ) and semi-infinite ${ }^{21}$ ) nuclear matter calculations, the temperature-dependent second-order gradient terms of the ETF model have been derived only very recently in short papers ${ }^{22-24}$ ). [In ref. ${ }^{22}$ ), only the functional of the free energy density $\mathscr{F}[\rho]$ for a local potential was derived; in ref. ${ }^{23}$ ), effective mass and spin-orbit contributions were also included.l In the present paper we shall give a comprehensive presentation of the ETF model at finite temperature and derive also the fourth-order gradient corrections to the free energy and entropy density functionals $\mathscr{F}[\rho]$ and $\sigma[\rho]$, respectively. We shall present tests of these functionals against quantum-mechanical calculations and discuss their application in variational calculations.

The paper is organized as follows. In sect. 2 we extend the Wigner-Kirkwood expansion of the Bloch density to finite temperature and derive the corresponding $\hbar$-expansions of the nuclear density, the free energy density and the entropy density in terms of the local (HF) potential $V(\boldsymbol{r})$ and its gradients, including also the effects of a variable effective nucleon mass and a spin-orbit potential up to second order in $\hbar$. In sect. 3 we show how the potential $V$, its gradients and the Fermi energy are eliminated in order to gain the functionals $\mathscr{F}[\rho]$ and $\sigma[\rho]$. In contrast to the kinetic energy density functional $\tau[\rho]$ at $T=0$, the functionals $\mathscr{F}[\rho]$ and $\sigma[\rho]$ are obtained at $T>0$ also in the classically forbidden region. In taking a careful limit $T \rightarrow 0$, we show that $\mathscr{F}[\rho]$ reduces to the old functional $\tau[\rho]$ at $T=0$ everywhere in space, thus strictly proving its hitherto only surmised validity at and outside the classical turning points. In sect. 4 we use the (deformed) harmonic-oscillator potential as a model to test the convergence of the semiclassical expansions against exact quantum-mechanical calculations at $T>0$. Sect. 5 is devoted to a discussion of the Euler variational equation obtained from the new TETF (i.e. temperature-dependent ETF) functionals in connection with a realistic Skyrme force. We show that this nonlinear fourth-order differential equation in the case of semi-infinite nuclear matter can be cast into a nonlinear second-order equation plus a simple quadrature. We discuss its numerical solutions for various temperatures and investigate the validity of variational calculations with parametrized trial densities. As an applica- 
tion, we calculate liquid-drop model parameters as functions of temperature. Some more technical details and involved formulae are presented in two appendices.

\section{The Wigner-Kirkwood expansion at $T>0$}

A convenient way to derive semiclassical expansions of energies or densities of a fermion system is to express them in terms of the partition function or the Bloch density and to use the Wigner-Kirkwood expansion ${ }^{25}$ ) of the latter in powers of $\hbar$. We will sketch this method only very briefly here and refer to the literature ${ }^{26}$ ) for the details.

One starts from the wave functions $\varphi_{i}(\boldsymbol{r})$ and eigenenergies $\varepsilon_{t}$ of a system of $N$ fermions ${ }^{\dagger}$ moving in an average potential $V(\boldsymbol{r})$ :

$$
\left\{-\frac{\hbar^{2}}{2 m} \Delta+V(\boldsymbol{r})\right\} \varphi_{l}(\boldsymbol{r})=\varepsilon_{\imath} \varphi_{l}(\boldsymbol{r}) .
$$

For the moment, we assume $V(\boldsymbol{r})$ to be local and velocity- and spin-independent. (Iater, we shall consider selfconsistent Hartree-Fock potentials and incorporate velocity- and spin-dependent parts.) All ground-state expectation values of this system can be calculated from the single-particle density matrix

$$
\rho\left(\boldsymbol{r}, \boldsymbol{r}^{\prime}\right)=\sum_{i=1}^{N} \varphi_{t}^{*}\left(\boldsymbol{r}^{\prime}\right) \varphi_{i}(\boldsymbol{r}),
$$

where the summation goes over the $N$ states with the lowest energies $\varepsilon_{i}$. The basic idea of the semiclassical approach $\left.{ }^{26}\right)$ is to express $\rho\left(\boldsymbol{r}, \boldsymbol{r}^{\prime}\right)$ in terms of the Bloch density matrix ${ }^{2 \prime}$ ) defined by

$$
C\left(\boldsymbol{r}, \boldsymbol{r}^{\prime} ; \boldsymbol{\beta}\right)=\sum_{\forall i} \varphi_{\iota}^{*}\left(\boldsymbol{r}^{\prime}\right) \varphi_{l}(\boldsymbol{r}) \mathrm{e}^{-\boldsymbol{\beta} \varepsilon_{i}} .
$$

Here the summation goes over the complete spectrum $\left\{\varphi_{l}\right\}$ (thus including an integral over continuum states if present). The density matrix $\rho\left(\boldsymbol{r}, \boldsymbol{r}^{\prime}\right)$, eq. (2.2), can be obtained from $C\left(\boldsymbol{r}, \boldsymbol{r}^{\prime} ; \beta\right)$, eq. (2.3), by

$$
\rho\left(\boldsymbol{r}, \boldsymbol{r}^{\prime}\right)=\mathscr{L}_{\lambda}^{-1}\left[\frac{1}{\beta} C\left(\boldsymbol{r}, \boldsymbol{r}^{\prime} ; \beta\right)\right],
$$

where $\lambda$ is the Fermi energy and the symbol $\mathscr{L}_{\lambda}^{-1}$ signifies an inverse Laplace transformation:

$$
\mathscr{L}_{\lambda}^{1}[f(\beta)]=\frac{1}{2 \pi i} \int_{c-l \infty}^{c+\infty} \mathrm{d} \beta \mathrm{e}^{\lambda \beta} f(\beta), \quad(c \text { real },>0) .
$$

Note that $\beta$ here is a complex mathematical variable and has nothing to do, in the present context, with any finite temperature. In fact, the inclusion of the factor $1 / \beta$

Throughout the formal parts of this paper, we consider only one kind of particle 
in eq. (2.4) and the use of $\lambda$ as the variable conjugate to $\beta$ automatically implies occupation of the states below the Fermi energy $\lambda$, and thus gives the ground-state density matrix. The quantity $\lambda$ is fixed by the particle number $N$ :

$$
N=\int \mathrm{d}^{3} r \rho(\boldsymbol{r}),
$$

where $\rho(\boldsymbol{r})$ is the local density distribution

$$
\rho(\boldsymbol{r})=\rho(\boldsymbol{r}, \boldsymbol{r}) .
$$

The kinetic energy density $\tau(\boldsymbol{r})$ is usually defined by

$$
\tau(\boldsymbol{r})=\left[\nabla_{r} \cdot \nabla_{r^{\prime}} \rho\left(\boldsymbol{r}, \boldsymbol{r}^{\prime}\right)\right]_{\boldsymbol{r}=\boldsymbol{r}^{\prime}} .
$$

Integrated quantities such as the particle number $N$ or the total single-particle energy $E_{\text {s.p. }}$,

$$
E_{\text {s.p. }}=\sum_{i=1}^{N} \varepsilon_{l},
$$

can also be expressed directly in terms of the partition function $Z(\beta)$

$$
Z(\beta)=\int \mathrm{d}^{3} r C(\boldsymbol{r}, \boldsymbol{r} ; \boldsymbol{\beta}),
$$

through the relations ${ }^{26}$ )

$$
\begin{aligned}
N & =\mathscr{L}_{\lambda}^{-1}\left[\frac{1}{\beta} Z(\beta)\right], \\
E_{\mathrm{sp} .} & =\lambda N-\mathscr{L}_{\lambda}^{-1}\left[\frac{1}{\beta^{2}} Z(\beta)\right] .
\end{aligned}
$$

Semiclassical expansions of $\rho(\boldsymbol{r}), \tau(\boldsymbol{r})$ or $E_{\text {s.p }}$ are now easily obtained by use of the $\hbar$-expansion of $C\left(\boldsymbol{r}, \boldsymbol{r}^{\prime} ; \beta\right)$ developed by Wigner, Kirkwood, Uhlenbeck and Beth $\left.^{25}\right)$. It has the form

$$
C_{\mathrm{WK}}\left(\boldsymbol{r}, \boldsymbol{r}^{\prime} ; \boldsymbol{\beta}\right)=C_{\mathrm{TF}}\left(\boldsymbol{r}, \boldsymbol{r}^{\prime} ; \beta\right)\left\{1+\sum_{n=1}^{\infty} \hbar^{n} \chi_{n}\left(\boldsymbol{r}, \boldsymbol{r}^{\prime} ; \boldsymbol{\beta}\right)\right\} .
$$

Here $C_{\mathrm{TF}}$ is the Thomas-Fermi approximation,

$$
C_{\mathrm{TF}}\left(\boldsymbol{r}, \boldsymbol{r}^{\prime} ; \boldsymbol{\beta}\right)=\left(\frac{m}{2 \pi \hbar^{2} \beta}\right)^{3 / 2} \mathrm{e}^{-\beta V\left(\left(\boldsymbol{r}+\boldsymbol{r}^{\prime}\right) / 2\right)} \mathrm{e}^{-m\left(\boldsymbol{r}-\boldsymbol{r}^{\prime}\right)^{2} / 2 \hbar^{2} \beta},
$$

and the $\chi_{n}\left(r, r^{\prime} ; \beta\right)$ contain powers of $\beta$ and gradients up to $n$th order of the potential $V(\boldsymbol{r})$. Inserting $C_{\mathrm{WK}}(2.13)$ into eq. (2.4), one obtains the $\hbar$-expansion of $\rho\left(\boldsymbol{r}, \boldsymbol{r}^{\prime}\right)$ and thus of all the local densities and expectation values of interest. [See ref. ${ }^{26}$ ) for detailed expressions up to fourth order in $\hbar$.] 


\subsection{TEMPERATURE DEPENDENCE OF THE BLOCH DENSITY}

We shall now extend the above formalism to a fermion system at finite temperature $T$, treated quantum-statistically as a grand canonical ensemble. The density matrix $\rho\left(\boldsymbol{r}, \boldsymbol{r}^{\prime}\right)$ eq. (2.2) is then replaced by

$$
\rho\left(\boldsymbol{r}, \boldsymbol{r}^{\prime}\right)=\sum_{\forall i} \varphi_{i}^{*}\left(\boldsymbol{r}^{\prime}\right) \varphi_{i}(\boldsymbol{r}) n_{i},
$$

where $n_{i}$ are the Fermi-Dirac occupation numbers

$$
n_{z}(T)=\left\{1+\exp \left(\frac{\varepsilon_{s}-\lambda}{T}\right)\right\}^{-1} .
$$

(We put the Boltzmann constant $k$ equal to one and measure $T$ in energy units.) As shown in ref. $\left.{ }^{12}\right)$, the density $\rho\left(\boldsymbol{r}, \boldsymbol{r}^{\prime}\right)$ eq. (2.15) can be formally obtained from the "cold" one, eq. (2.2), via a convolution of the $T=0$ spectral density (which is the Laplace inverse of $\left.C\left(r, r^{\prime} ; \beta\right)\right)$ with the function

$$
f_{T}(E)=\frac{1}{4 T} \cosh ^{-2}\left(\frac{E}{2 T}\right)
$$

Thus, due to the convolution theorem, the Bloch density at $T>0$ is a product,

$$
C_{T}\left(\boldsymbol{r}, \boldsymbol{r}^{\prime} ; \beta\right)=C_{0}\left(\boldsymbol{r}, \boldsymbol{r}^{\prime} ; \beta\right) \tilde{f}_{T}(\beta),
$$

of the Bloch density $C_{0}$ at $T=0$ (given by eq. (2.3)) and the (two-sided) Laplace transform $\tilde{f}_{T}(\beta)$ of the function $f_{T}(E)(2.17)$ :

$$
\tilde{f}_{T}(\beta)=\mathscr{L}_{\beta}\left[f_{T}(E)\right]=\int_{-\infty}^{+\infty} \mathrm{e}^{-\beta E} f_{T}(E) \mathrm{d} E=\frac{\pi \beta T}{\sin (\pi \beta T)} .
$$

Insertion of $C_{T}$, eq. (2.18), into eq. (2.4) automatically gives the density matrix $\rho\left(\boldsymbol{r}, \boldsymbol{r}^{\prime}\right)$, eq. (2.15), from which the densities $\rho(\boldsymbol{r})$ and $\tau(\boldsymbol{r})$ of the hot system are derived by means of eqs. (2.7), (2.8). The main integrated quantities of interest are the entropy

$$
S=-\sum_{\forall_{i}}\left[n_{t} \ln n_{t}+\left(1-n_{t}\right) \ln \left(1-n_{i}\right)\right]
$$

and the (single-particle) Helmholtz free energy

$$
F_{\text {s.p. }}=\sum_{\forall \varepsilon_{t}} n_{\imath}-T S \text {. }
$$

In order to relate these quantities to the Bloch density $C_{T}$, we introduce an entropy density $\boldsymbol{\sigma}(\boldsymbol{r})$,

$$
\sigma(\boldsymbol{r})=-\sum_{\forall i}\left|\varphi_{i}(\boldsymbol{r})\right|^{2}\left[n_{i} \ln n_{t}+\left(1-n_{i}\right) \ln \left(1-n_{i}\right)\right],
$$

and a free energy density $\mathscr{F}(\boldsymbol{r})$,

$$
\mathscr{F}(\boldsymbol{r})=\frac{\hbar^{2}}{2 m} \tau(\boldsymbol{r})+V(\boldsymbol{r}) \rho(\boldsymbol{r})-T \sigma(\boldsymbol{r}),
$$


So that

$$
S=\int \mathrm{d}^{3} r \sigma(\boldsymbol{r}), \quad F_{\text {s.p. }}=\int \mathrm{d}^{3} r \mathscr{F}(\boldsymbol{r})
$$

It is now relatively easy to verify ${ }^{12}$ ) that $\mathscr{F}(\boldsymbol{r})$ can be related to the local Bloch density by

$$
\mathscr{F}(\boldsymbol{r})=\lambda \rho(\boldsymbol{r})-\mathscr{L}_{\lambda}^{-1}\left[\frac{\lambda}{\beta^{2}} C_{T}(\boldsymbol{r}, \beta)\right]
$$

where

$$
C_{T}(\boldsymbol{r}, \beta)=C_{T}(\boldsymbol{r}, \boldsymbol{r} ; \beta)
$$

The entropy density then is simply obtained from $\mathscr{F}(\boldsymbol{r})$ by the canonical relation

$$
\boldsymbol{\sigma}(\boldsymbol{r})=-\left.\frac{\partial}{\partial T} \mathscr{F}(\boldsymbol{r})\right|_{\rho=\text { const }}=\mathscr{L}_{\lambda}^{-1}\left[\frac{1}{\beta^{2}} \frac{\partial}{\partial T} C_{T}(\boldsymbol{r}, \beta)\right] .
$$

In order to obtain the semiclassical expansions of the above densities, it is sufficient to replace the exact $T=0$ Bloch function $C_{0}\left(r, r^{\prime} ; \beta\right)$ by its WignerKirkwood expansion (2.13), hereby leaving the factor $\tilde{f}_{T}(\beta)$ in eq. (2.18) untouched. Before we give the results in the next subsection, we briefly mention the Wigner function defined by

$$
f(\boldsymbol{p}, \boldsymbol{q})=\int \mathrm{d}^{3} r \int \mathrm{d}^{3} r^{\prime} \rho\left(\boldsymbol{r}, \boldsymbol{r}^{\prime}\right) \mathrm{e}^{-(t / h) \boldsymbol{p} \cdot\left(\boldsymbol{r}-\boldsymbol{r}^{\prime}\right)} \delta\left(\boldsymbol{q}-\frac{\boldsymbol{r}+\boldsymbol{r}^{\prime}}{2}\right),
$$

which is often used to calculate local densities in terms of its moments in $\boldsymbol{p}$-space. For a local potential, one obtains from the Wigner-Kirkwood expansion up to second order in $\hbar$ :

$$
\begin{aligned}
f_{\mathrm{ETF}}(\boldsymbol{p}, \boldsymbol{q})= & n_{T}\left(\lambda-H_{\mathrm{cl}}\right)-\frac{\hbar^{2}}{8 m} \Delta V(\boldsymbol{q}) n_{T}^{\prime \prime}\left(\lambda-H_{\mathrm{cl}}\right) \\
& +\frac{\hbar^{2}}{24 m}\left\{[\nabla V(\boldsymbol{q})]^{2}+\frac{1}{m}\left(\boldsymbol{p} \cdot \nabla_{q}\right)^{2} V(\boldsymbol{q})\right\} n_{T}^{\prime \prime \prime}\left(\lambda-H_{\mathrm{cl}}\right),
\end{aligned}
$$

where $H_{\mathrm{cl}}$ is the classical Hamilton function

$$
H_{\mathrm{cl}}(\boldsymbol{p}, \boldsymbol{q})-\boldsymbol{p}^{2} / 2 m+V(\boldsymbol{q}),
$$

$n_{T}(E)$ is the Fermi function

$$
n_{T}(E)=\{1+\exp (-E / T)\}^{-1}=\int_{-\infty}^{E} f_{T}\left(E^{\prime}\right) \mathrm{d} E^{\prime},
$$

and $n_{T}^{\prime \prime}(E), n_{T}^{\prime \prime \prime}(E)$ are its derivatives. In the limit $T=0$, the function $n_{T}(E)$ becomes a step function and $f(\boldsymbol{p}, \boldsymbol{q})$ goes over into the form derived by Grammaticos and Voros $^{13}$ ). 


\section{2. $\hbar$-EXPANSION OF LOCAL DENSITIES}

Using the Wigner-Kirkwood expansion (2.13) of the Bloch density and performing the inverse Laplace transforms term by term, one gains the $\hbar$-expansions of the local densities defined above which only contain even powers of $\hbar$ :

$$
\begin{aligned}
& \rho_{\mathrm{ETF}}(\boldsymbol{r})=\rho_{\mathrm{TF}}(\boldsymbol{r})+\rho_{2}(\boldsymbol{r})+\rho_{4}(\boldsymbol{r})+\cdots, \\
& \mathscr{F}_{\mathrm{ETF}}(\boldsymbol{r})=\mathscr{F}_{\mathrm{TF}}(\boldsymbol{r})+\mathscr{F}_{2}(\boldsymbol{r})+\mathscr{F}_{4}(\boldsymbol{r})+\cdots, \\
& \sigma_{\mathrm{ETF}}(\boldsymbol{r})=\sigma_{\mathrm{TF}}(\boldsymbol{r})+\sigma_{2}(\boldsymbol{r})+\sigma_{4}(\boldsymbol{r})+\cdots,
\end{aligned}
$$

where the index shows the power of $\hbar$. To keep the formulae to a reasonable size, we shall here only present the TF and second-order expressions. (The fourth-order terms, as far as they will be of use, are given in appendix B for a local potential,) We include here the contributions from a variable effective mass in the one-body hamiltonian, such as it occurs in Hartree-Fock calculations with Skyrme-type effective nucleon-nucleon interactions ${ }^{28}$ ):

$$
\hat{H}=-\nabla \cdot \frac{\hbar^{2}}{2 m^{*}(\boldsymbol{r})} \nabla+V(\boldsymbol{r})
$$

(Spin-orbit contributions will be discussed at the end of this section.) We define an effective mass field $f(\boldsymbol{r})$ by

$$
f(\boldsymbol{r})=m / m^{*}(\boldsymbol{r})
$$

With this, the Wigner-Kirkwood expanded Bloch density becomes, up to order $\hbar^{2}$ [see refs. $\left.{ }^{16,29}\right)$ ],

$$
C_{\mathrm{WK}}(\boldsymbol{r}, \beta)=C_{\mathrm{TF}}(\boldsymbol{r}, \beta)\left\{1+\frac{\hbar^{2}}{24 m}\left[\beta b_{2}(\boldsymbol{r})-2 \beta^{2} d_{2}(\boldsymbol{r})+\beta^{3} f(\nabla V)^{2}\right]\right\},
$$

where

$$
C_{\mathrm{TF}}(r, \beta)=\left(\frac{m}{2 \pi \hbar^{2} \beta f}\right)^{3 / 2} \mathrm{e}^{-\beta \nu(r)}
$$

and

$$
b_{2}(\boldsymbol{r})=\frac{7}{4}(\nabla f)^{2} / f-5 \Delta f, \quad d_{2}(\boldsymbol{r})=f \Delta V-\frac{1}{2} \nabla f \cdot \nabla V .
$$

The well-known TF densities at finite temperature are ${ }^{19-21}$ )

$$
\begin{aligned}
& \rho_{\mathrm{TF}}(\boldsymbol{r})=A_{T}^{*} J_{1 / 2}\left(\eta_{0}\right), \\
& \mathscr{F}_{\mathrm{TF}}(\boldsymbol{r})=\lambda \rho_{\mathrm{TF}}(\boldsymbol{r})-\frac{2}{3} T A_{T}^{*} J_{3 / 2}\left(\eta_{0}\right), \\
& \sigma_{\mathrm{TF}}(\boldsymbol{r})=-\eta_{0} \rho_{\mathrm{TF}}(\boldsymbol{r})+{ }_{3}^{5} A_{T}^{*} J_{3 / 2}\left(\eta_{0}\right),
\end{aligned}
$$


where

$$
\begin{aligned}
& \eta_{0}=(\lambda-V(\boldsymbol{r})) / T \\
& A_{T}^{*}=\frac{1}{2 \pi^{2}}\left(\frac{2 m T}{\hbar^{2} f}\right)^{3 / 2}
\end{aligned}
$$

The second-order corrections become

$$
\begin{aligned}
& \rho_{2}(\boldsymbol{r})=\frac{1}{24} \frac{h^{2}}{2 m} A_{T}^{*}\left[\frac{b_{2}(\boldsymbol{r})}{T} J_{-1 / 2}\left(\eta_{0}\right)+\frac{d_{2}(\boldsymbol{r})}{T^{2}} J_{-3 / 2}\left(\eta_{0}\right)\right. \\
& \left.+\frac{3}{4 T^{3}}(\nabla V)^{2} J_{-5 / 2}\left(\eta_{0}\right)\right] \\
& \mathscr{F}_{2}(\boldsymbol{r})=\lambda \rho_{2}(\boldsymbol{r})+\frac{1}{12} \frac{\hbar^{2}}{2 m} A_{T}^{*}\left[-b_{2}(\boldsymbol{r}) J_{1 / 2}\left(\eta_{0}\right)\right. \\
& \left.+\frac{d_{2}(\boldsymbol{r})}{T} J_{-1 / 2}\left(\eta_{0}\right)+\frac{1}{4 T^{2}}(\nabla V)^{2} J_{-3 / 2}\left(\eta_{0}\right)\right], \\
& \sigma_{2}(\boldsymbol{r})=-\eta_{0} \rho_{2}(\boldsymbol{r})+\frac{1}{24} \frac{\hbar^{2}}{2 m} A_{T}^{*}\left[\frac{3 b_{2}(\boldsymbol{r})}{T} J_{\perp / 2}\left(\eta_{0}\right)\right. \\
& \left.-\frac{d_{2}(\boldsymbol{r})}{T^{2}} J_{-1 / 2}\left(\eta_{0}\right)+\frac{1}{4 T^{3}}(\nabla V)^{2} J_{-3 / 2}\left(\eta_{0}\right)\right] \text {. }
\end{aligned}
$$

In the above equations, $J_{\mu}\left(\eta_{0}\right)$ are the so-called Fermi integrals and their analytical continuations (for $\mu<-1$ ). They are defined and discussed in detail in appendix $A$.

The ETF expansion of the kinetic energy density $\tau(r)$, eq. (2.8), need not be given explicitly; it can easily be gained from the above results via eq. (2.23).

Note that the densities defined by eqs. (2.38)-(2.45) are analytical and finite functions of $\boldsymbol{r}$ in all space for any finite temperature $T>0$. This is different from the $T=0$ case, where the ETF expansions $\rho_{\mathrm{ETF}}(\boldsymbol{r})$ and $\tau_{\mathrm{ETF}}(\boldsymbol{r})$ are defined only inside the classically allowed region and identically zero outside; the $\hbar^{n}$ corrections with $n \geqslant 2$ even diverge at the classical turning points. This behaviour is easily recognized from the quantity $\eta_{0}$, eq. (2.41), which diverges with opposite signs for $T \rightarrow 0$ on either side of the turning points given by $\lambda=V(\boldsymbol{r})$. The known $T=0$ expressions for $\tau_{\mathrm{ETF}}(\boldsymbol{r})$ and $\rho_{\mathrm{ETF}}(\boldsymbol{r})$ [ref. $\left.{ }^{13,26}\right)$ ] are recovered for $T=0$ using the asymptotic values of the functions $J_{\mu}\left(\eta_{0}\right)$ discussed in appendix A. We will discuss the $T=0$ limil in more detail in subsect. 3.3 after evaluation of the density functionals $\mathscr{F}[\rho]$ and $\sigma[\rho]$.

The inclusion of a spin-orbit potential of the form ( $\sigma$ is the Pauli spin operator)

$$
\hat{V}_{\mathrm{s} . \mathrm{o} .}=-i \boldsymbol{W}(\boldsymbol{r}) \cdot(\nabla \times \boldsymbol{\sigma})
$$

up to order $\hbar^{2}$ is done exactly as in the $T=0$ case shown in ref. ${ }^{16}$ ). The lowest 
semiclassical contribution to the spin-orbit density, defined by ${ }^{28}$ )

$$
J(\boldsymbol{r})=-i\left[\left(\nabla_{r} \times \boldsymbol{\sigma}\right) \rho\left(\boldsymbol{r}, \boldsymbol{r}^{\prime}\right)\right]_{r=r^{\prime}},
$$

is of the form

$$
\boldsymbol{J}_{2}(\boldsymbol{r})=-\boldsymbol{W} \frac{2 m}{\hbar^{2}} \frac{1}{f} \rho_{\mathrm{TF}}(\boldsymbol{r}),
$$

where $\rho_{\mathrm{TF}}(\boldsymbol{r})$ now is given by eq. (2.38). The spin-orbit contribution of order $\hbar^{2}$ to the free energy density is

$$
\boldsymbol{\delta} \mathscr{F}_{2}^{\mathrm{s} o}(\boldsymbol{r})=-\frac{1}{2}(\boldsymbol{W})^{2} \frac{2 m}{\hbar^{2}} \frac{1}{f} \rho_{\mathrm{TF}}(\boldsymbol{r})
$$

\section{Density functionals for free energy and entropy}

Our aim is now to derive functionals which allow us to express the free energy and the entropy (and thus also the kinetic energy) in terms of the local density $\rho(\boldsymbol{r})$. According to the Hohenberg-Kohn theorem ${ }^{15}$ ) and its extension to fermion systems at finite temperature ${ }^{18}$ ), such functionals exist in principle. For an interacting fermion system, the exact functionals are not known. The difficulty of their determination is mainly due to (i) the presence of shell effects (at low temperatures), and (ii) the correlations. In our one-body problem we have no correlations to worry about, but still the shell effects make it hard to find a functional for the exact kinetic energy and the entropy.

On the other hand, for the average part of the kinetic energy, obtained e.g. by a Strutinsky-smoothing ${ }^{11}$ ), it is relatively easy to find an approximate functional. In fact, the gradient expansion of the kinetic energy density functional $\tau_{\mathrm{ETF}}[\rho]$ obtained from the ETF model at $T=0$ has been shown ${ }^{16}$ ) to reproduce the average kinetic energy in terms of the Strutinsky-averaged density $\tilde{\boldsymbol{\rho}}(\boldsymbol{r})$ to a very high degree of accuracy. This is not so surprising if one considers the well-established facts that (i) Strutinsky-averaging is semiclassical by nature and mathematically equivalent to the ETF $\hbar$-expansion ${ }^{26,30}$ ), and (ii) a microscopically Strutinsky-averaged fermion system can be formulated variationally ${ }^{12}$ ) and thus the Hohenberg-Kohn theorem applies to the averaged energy as a functional of the averaged density $\tilde{\rho}(\boldsymbol{r})$.

It is thus to be expected that the extension of the ETF model to finite temperature should lead to functionals which may be useful for excited nuclear systems. In particular, at temperatures $T \geq 2.5$ to $3 \mathrm{MeV}$, where the shell effects are washed out $^{5-7}$ ), one may hope that these functionals become valid for the exact quantum-mechanical free energy $F_{\text {s.p. }}$ and entropy $S$. This has already been made evident ${ }^{24}$ ) by comparing the results of density variational calculations with the approximate $T>0$ functionals against those of Hartree-Fock calculations. In this section we shall briefly resume the derivation of the ETF gradient corrections at 
$T>0$ up to second order ${ }^{22-24}$ ) and give also, for the first time, the fourth-order corrections for the case of a local potential. The new functionals will then be tested in fully quantum-mechanical calculations with a model potential in sect. 4 .

\subsection{SECOND-ORDER FUNCTIONALS FOR A SKYRME-TYPE NONLOCAL POTENTIAL}

Following the method used in ref. ${ }^{23}$ ), we shall now eliminate the potential and its gradients, as well as the chemical potential $\lambda$, from the ETF densities given in subsect. 2.2. To lowest order, i.e. in the TF approximation, this elimination can only be done by inverting eq. (2.38) numerically: for any given density one finds $\eta_{0}$ and inserts it into eqs. (2.39), (2.40) to obtain the free energy and the entropy density. (Note that in variational calculations with Skyrme-type interactions, the effective mass function $f(\boldsymbol{r})$, eq. (2.34), is also a function of the density $\rho$.)

In order to incorporate the gradient corrections of the $h$-expansion, we now define as the full density $\rho(\boldsymbol{r})$ the sum of all terms in eq. (2.32) up to the desired order in $\hbar$ :

$$
\rho(\boldsymbol{r})=\rho_{\mathrm{ETF}}(\boldsymbol{r})=\boldsymbol{\rho}_{\mathrm{TF}}(\boldsymbol{r})+\rho_{2}(\boldsymbol{r})+\rho_{4}(\boldsymbol{r})+\cdots .
$$

This is the density as functionals of which we want to express $\mathscr{F}_{\mathrm{ETF}}(\boldsymbol{r})$ and $\sigma_{\mathrm{ETF}}(\boldsymbol{r})$, and which is treated variationally in the practical applications.

The salient point ${ }^{23}$ ) is now to define a new parameter $\eta$ by the relation

$$
\rho(\boldsymbol{r})=A_{T}^{*} J_{1 / 2}(\eta) .
$$

(The function $f(\boldsymbol{r})$ occurring in $A_{T}^{*}$, eq. (2.42), must hereby also be taken as function of $\rho(\boldsymbol{r})$.) Note that $\eta$ is different from $\eta_{0}$ eq. (2.41), because the higher-order terms are incorporated in $\rho(\boldsymbol{r})$ through eq. (3.1). Next we formally expand $\eta$ in a series

$$
\eta=\eta_{0}+\eta_{2}+\cdots,
$$

where $\eta_{0}$ is given by eq. (2.41) and the $\eta_{n}$ with $n>0$ are of order $\hbar^{n}$ relative to $\eta_{0}$. We now expand the right-hand side of eq. (3.2) around $\eta_{0}$ to obtain

$$
\rho=A_{T}^{*} J_{1 / 2}\left(\eta_{0}\right)+\eta_{2} A_{T}^{* \frac{1}{2}} J_{-1 / 2}\left(\eta_{0}\right)+\cdots .
$$

(Hereby we used the relation (A.5) in appendix A.) By comparison of eqs. (3.4) and (3.1) term by term - the lowest terms are identical by construction - it is now possible to express $\eta_{2}, \eta_{4}, \ldots$ successively through $\rho_{2}(r), \rho_{4}(\boldsymbol{r}), \ldots$ (See eq. (B.10) in appendix B for the resulting $\eta_{2}$.)

In the next step we expand $\mathscr{F}_{\mathrm{ETF}}(\boldsymbol{r})$ eq. (2.32), which is given in terms of $\eta_{0}$, around $\eta$. For this purpose it is useful to reorganize the series (2.32) for $\mathscr{F}_{\mathrm{ETF}}(\boldsymbol{r})$ including the terms $\lambda \rho_{n}(\boldsymbol{r})$ occurring in $\mathscr{F}_{n}(\boldsymbol{r})$ - see eqs. (2.39), (2.44) - into the TF expression; using also eq. (2.41) to eliminate $\lambda$ we thus redefine

$$
\begin{aligned}
\tilde{F}_{\mathrm{TF}}\left(\boldsymbol{r}, \eta_{0}\right) & =\rho(\boldsymbol{r})\left(\boldsymbol{T} \eta_{0}+\boldsymbol{V}\right)-\frac{2}{3} A_{T}^{*} I J_{3 / 2}\left(\eta_{0}\right), \\
\tilde{\mathscr{F}_{n}}\left(\boldsymbol{r}, \eta_{0}\right) & =\mathscr{F}_{n}(\boldsymbol{r})-\lambda \rho_{n}(\boldsymbol{r}) \quad(n=2,4, \ldots),
\end{aligned}
$$


so that

$$
\mathscr{F}_{\mathrm{ETF}}\left(\boldsymbol{r}, \eta_{0}\right)=\tilde{\mathscr{F}}_{\mathrm{TF}}+\tilde{\mathscr{F}}_{2}+\tilde{\mathscr{F}}_{4}+\cdots
$$

It now requires just some algebra to expand $\mathscr{F}_{\mathrm{ETF}}\left(\boldsymbol{r}, \eta_{0}\right)$ around $\eta$ and to insert the $\eta_{n}(n>0)$ found previously. (This is shown in appendix $\mathrm{B}$ for the case of a local potential.)

The final step is to express the gradients of the potential $V(\boldsymbol{r})$ in terms of gradients of the density $\rho(\boldsymbol{r})$. This is done by taking the corresponding derivatives of eq. (3.1), not forgetting that $\nabla \eta_{0}$ equals $-\nabla V / T$ etc., re-expanding those expressions around $\eta$, and inverting the resulting equations. The whole procedure, if carried through consistently up to order $\hbar^{4}$, requires some tedious algebra but is straightforward. We present here the result up to second order for a one-body hamiltonian of the form (2.33) with variable effective mass, including also a spin-orbit term, eq. (2.46). The functional for the free energy density becomes ${ }^{23}$ )

$$
\mathscr{F}_{\text {TETF }}[\rho]=\mathscr{F}_{\text {TF }}[\rho]+\mathscr{F}_{2}[\rho]+\mathscr{\mathscr { F }}_{4}[\rho]+\cdots
$$

with

$$
\begin{aligned}
& \mathscr{F}_{\mathrm{TF}}[\rho]=V \rho+T \eta \rho-\frac{2}{3} T A_{T}^{*} J_{3 / 2}(\eta), \\
& \mathscr{F}_{2}[\rho]=\frac{\hbar^{2}}{2 m}\left\{\zeta(\eta) f \frac{(\nabla \rho)^{2}}{\rho}+\right. {\left[\frac{2}{4} \zeta(\eta)-\frac{7}{48}\right] \rho \frac{(\nabla f)^{2}}{f} } \\
&\left.+\left[3 \zeta(\eta)-\frac{5}{12}\right] \nabla \rho \cdot \nabla f\right\}-\frac{1}{2}(W)^{2} \frac{2 m}{\hbar^{2}} \frac{1}{f} \rho .
\end{aligned}
$$

(We leave out a term proportional to $\Delta \rho$ which does not contribute to the total free energy.) Hereby we have defined the coefficient $\zeta(\eta)$ as

$$
\zeta(\eta)=-\frac{1}{6} J_{1 / 2}(\eta) \frac{\mathrm{d}}{\mathrm{d} \eta}\left[\frac{1}{J_{-1 / 2}(\eta)}\right]=-\frac{J_{1 / 2}(\eta) J_{-3 / 2}(\eta)}{12 J_{-1 / 2}^{2}(\eta)} .
$$

Note that $\zeta(\eta)$ depends on $\rho$ and the temperature $T$ through eq. (3.2). It is, however, a universal function of $\eta$ which can be computed once and for all. The coefficient $\zeta(\eta)$ was also derived independently in a different context by a completely different method $^{22}$ ); the functional $\mathscr{F}_{2}[0]$ given there corresponds to a local potential (thus $f \equiv 1$ and $\boldsymbol{W} \equiv 0$ ) and contains only the first term of our above result ${ }^{\dagger}$. A rough approximation (to within less than $3 \%$ for all values of $\eta$ ) is ${ }^{23}$ )

$$
\zeta(\eta)=\frac{1}{36}\left[1+2 /\left(1+\mathrm{e}^{\eta}\right)^{1 / 2}\right] .
$$

A better numerical approximation may be found in ref. ${ }^{22}$ ).

$\dagger$ Eq. (14) of ref. ${ }^{22}$ ) contains a trivial misprint. 
The functional for the entropy density

$$
\boldsymbol{\sigma}_{\mathrm{TETF}}[\rho]-\boldsymbol{\sigma}_{\mathrm{TF}}[\rho]+\sigma_{2}[\rho]+\sigma_{4}[\rho]+\cdots
$$

is found most easily using

$$
\sigma[\rho]=-\left.\frac{\partial}{\partial T} \mathscr{F}[\rho]\right|_{\lambda, \rho=\text { const }} .
$$

We obtain

$$
\begin{aligned}
\sigma_{\mathrm{TF}}[\rho] & =\frac{5}{3} A_{T}^{*} J_{3 / 2}(\eta)-\eta \rho \\
\sigma_{2}[\rho] & =-\frac{1}{T} \frac{\hbar^{2}}{2 m} \nu(\eta)\left\{f \frac{(\nabla \rho)^{2}}{\rho}+\frac{9}{4} \rho \frac{(\nabla f)^{2}}{f}+3 \nabla \rho \cdot \nabla f\right\} .
\end{aligned}
$$

The coefficient $\nu(\eta)$ is defined by (see also eqs. (B.20), (B.24))

$$
\nu(\eta)=\left.T \frac{\partial}{\partial T} \zeta(\eta)\right|_{\rho}=-3 \frac{J_{1 / 2}(\eta)}{J_{-1 / 2}(\eta)} \frac{\mathrm{d} \zeta(\eta)}{\mathrm{d} \eta} .
$$

The kinetic energy density functional need not be given explicitly; it is simply obtained from

$$
\frac{\hbar^{2}}{2 m} f \tau[\rho]=\mathscr{F}[\rho]+T \sigma[\rho]-V \rho
$$

\subsection{FOURTH-ORDER FUNCTIONALS FOR LOCAL POTENTIALS}

To carry the variable effective mass and the spin-orbit corrections to fourth order by hand would require a formidable amount of work which is better left to a computer, as was done for the $T=0$ functional for $\tau[\rho]$ by Grammaticos and Voros ${ }^{13}$ ). We have done the case of a purely local potential; some intermediate results are given in the appendix B. The final fourth-order gradient corrections are, after partial integrations,

$$
\begin{aligned}
& \mathscr{F}_{4}[\rho]=\frac{1}{T}\left(\frac{\hbar^{2}}{2 m}\right)^{2}\left[\theta_{1}(\eta) \frac{(\Delta \rho)^{2}}{\rho}+\theta_{2}(\eta) \frac{\Delta \rho(\nabla \rho)^{2}}{\rho^{2}}+\theta_{3}(\eta) \frac{(\nabla \rho)^{4}}{\rho^{3}}\right] \\
& \sigma_{4}[\rho]=\frac{1}{T^{2}}\left(\frac{\hbar^{2}}{2 m}\right)^{2}\left[\chi_{1}(\eta) \frac{(\Delta \rho)^{2}}{\rho}+\chi_{2}(\eta) \frac{\Delta \rho(\nabla \rho)^{2}}{\rho^{2}}+\chi_{3}(\eta) \frac{(\nabla \rho)^{4}}{\rho^{3}}\right] .
\end{aligned}
$$

The coefficients $\theta_{i}(\eta)$ and $\chi_{i}(\eta)$ are again universal functions of $\eta$ which are given in appendix $B$ in terms of the $J_{\mu}(\eta)$.

+ Eq. (55) of ref. ${ }^{23}$ ) is in error. 


\section{3. $T=0$ LIMIT OF THE TETF FUNCTIONALS}

It is interesting to investigate the $T=0$ limit of the new TETF functionals for the following reason. In the standard derivation ${ }^{13,16}$ ) of the ETF functionals at $T=0$, the classically forbidden region is not accessible. As we mentioned in subsect. 2.2 , the densities $\tau_{\mathrm{ETF}}(\boldsymbol{r})$ and $\rho_{\mathrm{ETF}}(\boldsymbol{r})$ diverge at the classical turning points for $T=0$ and are identically zero outside. The functional $\tau_{\text {ETF }}[\rho]$ derived at $T=0$ is therefore a priori only justified inside the classically allowed region. It was nevertheless used in innumerable cases under the assumption that it could be analytically continued to the whole space.

From our theory at $T>0$, it has become possible for the first time to verify this analytical continuation ${ }^{24}$ ). [See also ref. ${ }^{10}$ ) for an explicit discussion.] The reason is simply that one has no turning point problem at $T>0$; the densities $\rho_{\text {ETF }}(\boldsymbol{r}), \mathscr{F}_{\text {ETF }}(\boldsymbol{r})$, etc. are all analytical and therefore the TETF functionals given above are strictly valid in all space.

Using the fact that $\rho$, eq. (3.2), is a monotonously increasing function of $\eta$ and of temperature $T$, and using the asymptotic behaviour of $J_{1 / 2}(\eta)$ for large $\eta$ (see eq. (A.11)), it is seen ${ }^{10.24}$ ) that for any finite density $\rho$, the parameter $\eta$ goes to infinity in the limit $T \rightarrow 0$ like $1 / T$ :

$$
\eta \underset{T \rightarrow 0}{\longrightarrow} \frac{1}{T} \frac{\hbar^{2}}{2 m} f\left(3 \pi^{2} \rho\right)^{2 / 3}+\mathrm{O}(T) .
$$

From the asymptotic values of the coefficients $\zeta(\eta), \nu(\eta), \theta_{i}(\eta)$ and $\chi_{i}(\eta)$ for large $\eta$ given in table 4 in appendix $B$, and using eq. (3.21) it is readily verified that the functional $\mathscr{F}_{\text {TETF }}[\rho]$ goes over to the kinetic energy density functional known ${ }^{10,13,16}$ ) at $T=0$

$$
\mathscr{F}_{\mathrm{TETF}}[\rho] \underset{T \rightarrow 0}{\longrightarrow} \frac{\hbar^{2}}{2 m} f_{\tau}^{T=0}[\rho]+V \rho,
$$

and the entropy density $\sigma_{\text {TETF }}[\rho]$ goes to zero like $T$. This holds at any point in space where the density is finite, even if it is arbitrarily small.

A note on the sixth- and higher-order gradient corrections is in place here. From dimensional considerations, the correction $\mathscr{F}_{6}[\rho]$ must be of the form

$$
\mathscr{F}_{6}[\rho]=\frac{1}{T^{2}}\left(\frac{\hbar^{2}}{2 m}\right)^{3}\left[\psi_{1}(\eta) \frac{(\Delta \rho)^{3}}{\rho^{2}}+\psi_{2}(\eta) \frac{(\Delta \rho)^{2}(\nabla \rho)^{2}}{\rho^{3}}+\cdots\right]
$$

it will give a finite contribution to the free energy at $T>0$. The coefficients $\psi_{1}(\eta), \psi_{2}(\eta), \ldots$ will in the limit $T \rightarrow 0$ go like $\eta^{-2}$ :

$$
\Psi_{l}(\eta) \underset{T \rightarrow 0}{\rightarrow} \eta^{-2} \propto T^{2}\left(2 m / \hbar^{2}\right)^{2} \rho^{-4 / 3} .
$$

Hence the correction $\tau_{6}[\rho]$ (and also all the higher-order terms) to the kinetic energy functional at $T=0$ will diverge for any realistic density which goes exponentially to 
zero for large distances, and will give an infinite contribution to the kinetic energy. Since the ETF density gradient expansions are to be understood as asymptotic series, the functional $\tau_{\mathrm{ETF}}[\rho]$ at $T=0$ must therefore be truncated after the fourth-order term, as discussed before ${ }^{10,16}$ ).

\section{Test calculations with harmonic-oscillator wave functions}

In this section, the semiclassical expansions of the free energy density $\mathscr{F}$, kinetic energy density $\tau$ and entropy density $\sigma$ as functionals of the local density $\rho$ will be tested against their quantum-mechanical analogues defined directly in terms of wave functions. According to the theorem of Hohenberg and Kohn ${ }^{15,18}$ ) these functionals do not depend on the particular form of the potential, as long as it is local. As a simple approximation to a selfconsistent mean field, the harmonic oscillator is frequently used because of the simple form of its eigenfunctions. We shall use it here as a testing model for which the densities $\rho(\boldsymbol{r}), \mathscr{F}(\boldsymbol{r}), \tau(\boldsymbol{r})$ and $\boldsymbol{\sigma}(\boldsymbol{r})$ can be calculated analytically. Since shell effects disappear at excitation energies corresponding to a nuclear temperature of the order of $3 \mathrm{MeV}$, the functionals derived in sect. 3 can be used for $T \geq 3 \mathrm{MeV}$ with the exact quantum-mechanical densities. For high enough temperatures $(T \geqslant 5 \mathrm{MeV})$, these show indeed a completely smooth shape without quantum oscillations. We shall also investigate the Wigner-Kirkwood expansion of the partition function at finite temperature and the semiclassical expansions of the kinetic energy and the entropy, which can be derived from it analytically.

We choose the harmonic-oscillator potential to be axially deformed and write in cylindrical coordinates with $r=\sqrt{x^{2}+y^{2}}$,

$$
V(r, z)=\frac{1}{2} m\left(\omega_{\perp}^{2} r^{2}+\omega_{z}^{2} z^{2}\right) .
$$

The frequencies are chosen as

$$
\omega_{\perp}=\omega_{0} q^{1 / 3}, \quad \omega_{z}=\omega_{0} q^{-2 / 3},
$$

so that the axis ratio $q=\omega_{\perp} / \omega_{z}$ can be used as a deformation parameter. The eigenvalues $\varepsilon_{\imath}$ and eigenfunctions $\varphi_{\imath}$ of this potential are standard and need not be given here. The density distribution

$$
\rho(\boldsymbol{r})=\sum_{i}\left|\varphi_{i}(r)\right|^{2} n_{t}
$$

and the kinetic energy density

$$
\tau(\boldsymbol{r})=\sum_{l}\left|\nabla \varphi_{l}(\boldsymbol{r})\right|^{2} n_{l},
$$

as well as the entropy density $\sigma(\boldsymbol{r})$, eq. (2.22), are then readily computed with the Fermi occupation numbers $n$, given in eq. (2.16). 


\subsection{THE WIGNER-KIRKWOOD EXPANSION AT FINITE TEMPERATURE}

Beforc we test the density functionals derived in sect. 3, let us first study integrated quantities such as the free energy $F$ or the entropy $S$ in the WignerKirkwood expansion. For this purpose we write $F$ and $S$ as inverse Laplace transforms as done in eqs. (2.25) and (2.27), but replacing the Bloch density $C_{T}(r, \beta)$ by the partition function $Z_{T}(\beta)$. For the harmonic-oscillator potential (4.1) this is given by (including a spin factor 2 )

$$
Z_{T}(\beta)=Z_{0}(\beta) \tilde{f}_{T}(\beta)=\frac{1}{4 \sinh ^{2}\left(\frac{1}{2} \beta h \omega_{\perp}\right) \sinh \left(\frac{1}{2} \beta \hbar \omega_{z}\right)} \tilde{f}_{T}(\beta)
$$

with $\tilde{f}_{T}(\beta)$ defined by eq. (2.19). The partition function $Z_{0}(\beta)$ can be expanded in powers of $\hbar$ as done in ref. ${ }^{26}$ ) for spherical shapes

$$
\begin{aligned}
& Z_{0}^{\text {s.c. }}(\beta)=\frac{2}{\left(\beta \hbar \omega_{0}\right)^{3}}\{1-\frac{\left(\beta \hbar \omega_{0}\right)^{2}}{24}\left(2 q^{2 / 3}+q^{-4 / 3}\right) \\
&\left.1 \cdot \frac{\left(\beta \hbar \omega_{0}\right)^{4}}{5760}\left(24 q^{4 / 3}+20 q^{-2 / 3}+7 q^{-8 / 3}\right)\right\} .
\end{aligned}
$$

The total free energy $F$ is obtained as

$$
F=\lambda N-\mathscr{L}_{\lambda}^{-1}\left[\frac{1}{\beta^{2}} Z_{T}(\beta)\right]=\lambda N-\mathscr{L}_{\lambda}^{-1}\left[\frac{1}{\beta^{2}} Z_{0}(\beta) \tilde{f}_{T}(\beta)\right] .
$$

Similarly the entropy $S$ can be expressed as

$$
S=\frac{\partial}{\partial T} \mathscr{L}_{\lambda}^{-1}\left[\frac{1}{\beta^{2}} Z_{0}(\beta) \tilde{f}_{T}(\beta)\right]=\frac{1}{T} \mathscr{L}_{\lambda}^{-1}\left[\frac{Z_{0}(\beta)}{\beta} \frac{\partial \tilde{f}_{T}}{\partial \beta}\right] .
$$

In these equations the chemical potential $\lambda$ is fixed by eq. (2.11) to yield the correct particle number.

Inserting the $h$-expansion of the partition function $Z_{0}$ eq. (4.6), one can express $N, F$ and $S$ through the Fermi integrals $J_{\mu}, \tilde{J}_{\mu}$ given in appendix A. For spherical shapes one obtains ${ }^{\dagger}$ (up to order $\hbar^{4}$ )

$$
\begin{aligned}
N & =\left(\frac{T}{\hbar \omega_{0}}\right)^{3} J_{2}\left(\frac{\lambda}{T}\right)-\frac{T}{4 \hbar \omega_{0}} J_{0}\left(\frac{\lambda}{T}\right)+\frac{17}{960} \frac{\hbar \omega_{0}}{T} \tilde{J}_{-2}\left(\frac{\lambda}{T}\right), \\
F_{\mathrm{WK}} & =\lambda N-\frac{1}{3} \frac{T^{4}}{\left(\hbar \omega_{0}\right)^{3}} J_{3}\left(\frac{\lambda}{T}\right)+\frac{1}{4} \frac{T^{2}}{\hbar \omega_{0}} J_{1}\left(\frac{\lambda}{T}\right)-\frac{17}{960} \hbar \omega_{0} \tilde{J}_{-1}\left(\frac{\lambda}{T}\right), \\
S_{\mathrm{WK}} & =\frac{\partial}{\partial T}\left\{\frac{1}{3} \frac{T^{4}}{\left(\hbar \omega_{0}\right)^{3}} J_{3}\left(\frac{\lambda}{T}\right)-\frac{1}{4} \frac{T^{2}}{\hbar \omega_{0}} J_{1}\left(\frac{\lambda}{T}\right)+\frac{17}{960} \hbar \omega_{0} \tilde{J}_{-1}\left(\frac{\lambda}{T}\right)\right\} .
\end{aligned}
$$

$\uparrow$ For economical reasons the results are given here only for spherical shapes. The extension to the deformed case is very easy and follows directly from the partition function (4.6). 
For not too light nuclei the argument $(\lambda / T)$ of the Fermi integrals is much larger than unity for nuclear temperatures up to about $10 \mathrm{MeV}$. This enables us to use the asymptotic expansion (A.11) of $J_{\mu}(\eta)$ for $\eta \gg 1$ to obtain

$$
\begin{aligned}
N= & \frac{1}{3}\left(\frac{\lambda}{\hbar \omega_{0}}\right)^{3}-\frac{1}{4} \frac{\lambda}{\hbar \omega_{0}}+\frac{\pi^{2}}{3} \lambda \frac{T^{2}}{\left(\hbar \omega_{0}\right)^{3}}+\frac{17}{960} \frac{\hbar \omega_{0}}{T} \frac{\mathrm{e}^{-\lambda / T}}{\left[1+\mathrm{e}^{-\lambda / T}\right]^{2}} \\
F_{\mathrm{WK}}= & \frac{1}{4} \frac{\lambda^{4}}{\left(\hbar \omega_{0}\right)^{3}}-\frac{1}{8} \frac{\lambda^{2}}{\hbar \omega_{0}}+\frac{\pi^{2}}{6} \frac{\lambda^{2} T^{2}}{\left(\hbar \omega_{0}\right)^{3}}+\frac{\pi^{2}}{24} \frac{T^{2}}{\hbar \omega_{0}}-\frac{7 \pi^{4}}{180} \frac{T^{4}}{\left(\hbar \omega_{0}\right)^{3}} \\
& +\frac{17}{960} \frac{\hbar \omega_{0}}{1+\mathrm{e}^{-\lambda / T}}\left[\frac{\lambda}{T} \frac{\mathrm{e}^{-\lambda / T}}{1+\mathrm{e}^{-\lambda / T}}-1\right] \\
S_{\mathrm{WK}}= & \frac{\pi^{2}}{3}\left(\frac{\lambda}{\hbar \omega_{0}}\right)^{2} \frac{T}{\hbar \omega_{0}}-\frac{\pi^{2}}{12} \frac{T}{\hbar \omega_{0}}+\frac{7 \pi^{4}}{45}\left(\frac{T}{\hbar \omega_{0}}\right)^{3}-\frac{17}{960} \frac{\lambda \hbar \omega_{0}}{T^{2}} \frac{\mathrm{e}^{-\lambda / T}}{\left[1+\mathrm{e}^{-\lambda / T}\right]^{2}}
\end{aligned}
$$

(Note that the expansion (A.11) is limited to a finite number of terms for the $J_{\mu}(\eta)$ with non-negative integer index $\mu$.)

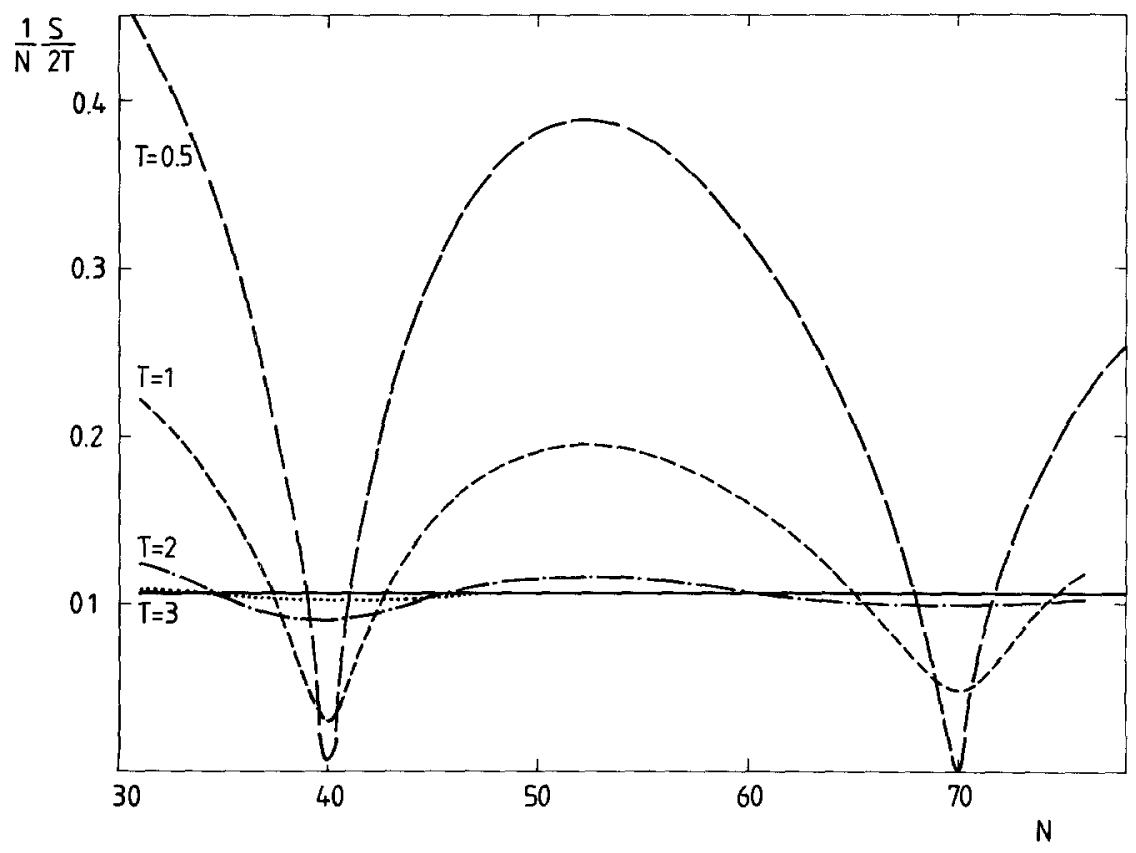

Fig. 1. Entropy versus particle number at various temperatures (in $\mathrm{MeV}$ ). Dashed and dotted lines: exact quantum-mechanical results. Solld line: result of the semiclassical Wigner-Kirkwood expansion. The temperature dependence of the semiclassical quantity shown is too small to be visible on the figure. 
TABLE 1

Entropy $S$ and free energy $F$ of $N=70$ fermions in a spherical harmonic-oscillator potential at different temperaturcs $T$; the contributions at different orders in the $\hbar$-expansions (4.11) and (4.12) are shown and their sums (WK) are compared to the exact quantum-mechanical values (ex)

\begin{tabular}{cccccccccc}
\hline $\begin{array}{c}T \\
{[\mathrm{MeV}]}\end{array}$ & $S_{\mathrm{TF}}$ & $S_{2}$ & $S_{\mathrm{WK}}$ & $S_{\mathrm{ex}}$ & $\begin{array}{c}F_{\mathrm{TF}} \\
{[\mathrm{MeV}]}\end{array}$ & $\begin{array}{c}F_{2} \\
{[\mathrm{MeV}]}\end{array}$ & $\begin{array}{c}F_{4} \\
{[\mathrm{MeV}]}\end{array}$ & $\begin{array}{c}F_{\mathrm{WK}} \\
{[\mathrm{MeV}]}\end{array}$ & $\begin{array}{c}F_{\mathrm{ex}} \\
{[\mathrm{MeV}]}\end{array}$ \\
\hline 0 & & & & & 2534.48 & -35.37 & -0.14 & 2498.97 & 2487.24 \\
1 & 14.91 & -0.10 & 14.81 & 6.67 & 2526.92 & -35.21 & -0.14 & 2491.57 & 2485.89 \\
3 & 44.43 & -0.31 & 44.12 & 43.86 & 2466.63 & -33.96 & -0.14 & 2432.53 & 2432.41 \\
5 & 73.06 & -0.52 & 72.54 & 72.54 & 2347.31 & -31.49 & -0.14 & 2315.68 & 231568 \\
\hline
\end{tabular}

To test the convergence of the semiclassical expansions (4.11) and (4.12) we compare the results thus obtained with the exact values for $F$ and $S$ as calculated microscopically with eqs. (2.20), (2.21). As an illustration we show in fig. 1 the entropy $S$. A very similar comparison can be made when investigating e.g. the excitation energy $E^{*}$. A pronounced shell structure is observed in the quantum-mechanical results at low excitations; it is washed out as the temperature increases. For $T \gtrsim 3 \mathrm{MeV}$ the exact and the semiclassical results coincide ${ }^{\dagger}$ showing that for such temperatures the Wigner-Kirkwood expansion reproduces the quantum-mechanical results. To study the convergence of the semiclassical expansion, we give in table 1 for $N=70$ particles the contributions at different orders in $\hbar$ and compare their sum to the exact results. The rapid convergence known for the $T=0$ case $\left.^{26}\right)$ is recovered here.

\subsection{TEST OF THE TETF DENSITY FUNCTIONALS}

We shall now test the functionals $\mathscr{F}_{\mathrm{TETF}}[\rho]$ and $\sigma_{\mathrm{TETF}}[\rho]$ derived in sect. 3 by inserting the exact density $\rho(\boldsymbol{r})$, eq. (4.3), into them and comparing the results with the corresponding quantities evaluated directly from the wave functions. In fig. 2 we show the kinetic energy density and entropy densities for two different temperatures. Rather than the kinetic energy density $\tau(\boldsymbol{r})$ as defined in eq. (4.4) we have drawn the equivalent quantity

$$
\bar{\tau}(\boldsymbol{r})=\tau(\boldsymbol{r})-\frac{1}{4} \Delta \boldsymbol{\rho}
$$

which gives identical kinetic energies but shows almost no quantum oscillations even at zero temperature. Instead of using the full $\mathscr{F}_{4}[\mu]$ as given in appendix $B$ and the corresponding $\sigma_{4}[\rho]$ we have included the fourth-order corrections in the form of eqs. (3.19), (3.20) which give the same contribution to the total $F_{4}$ and $S_{4}$, respectively. The distributions obtained are very close to the exact ones. It might

\footnotetext{
$\ddagger$ The temperature dependence of the semiclassical quantities is too small to be visible on the figure.
} 

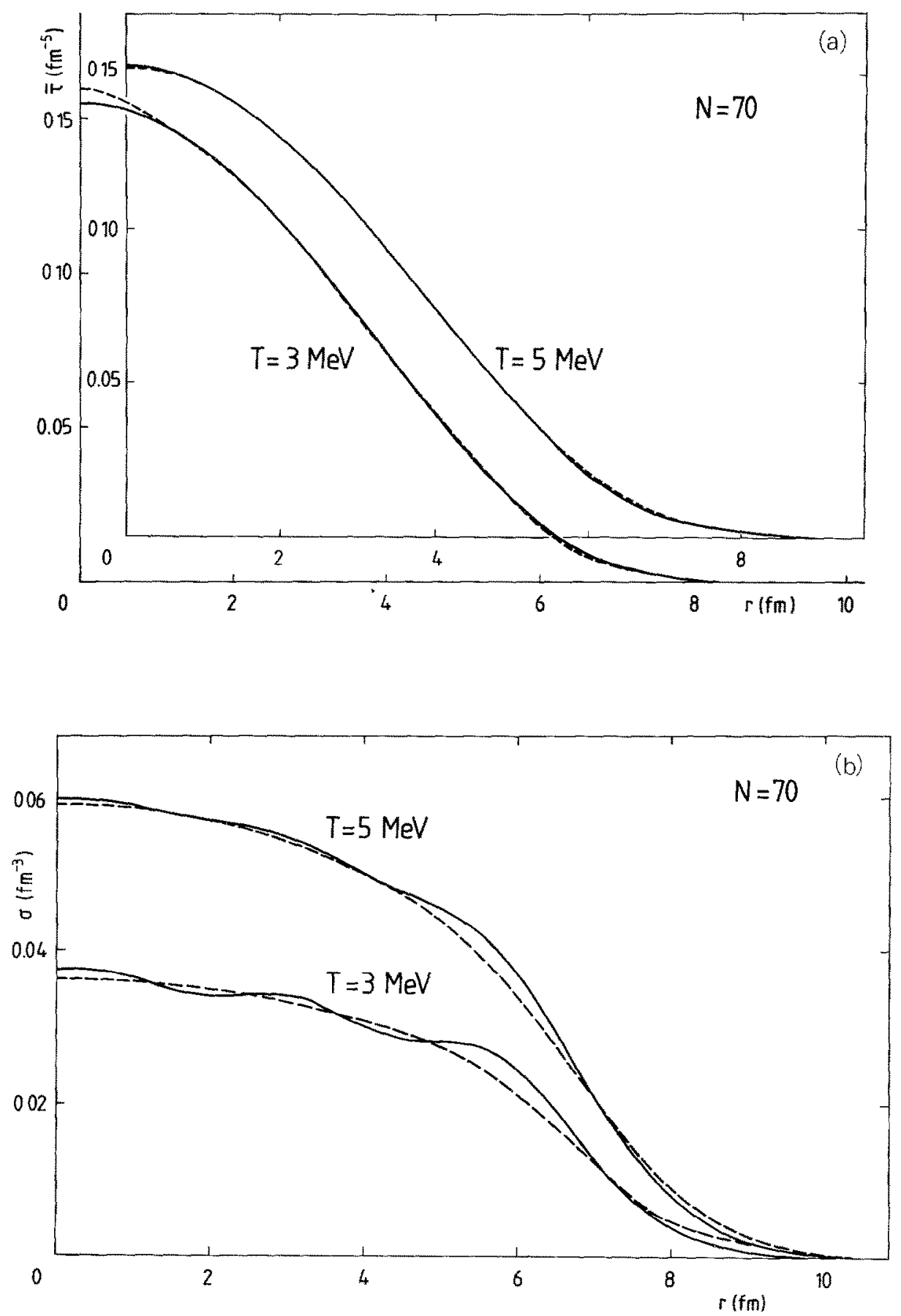

Fig. 2. Kinetic energy density $\tau(r)$ (a) and entropy density $\sigma(r)$ (b) for two different temperatures. The exact quantum-mechanical distributions (full lines) are compared with the semiclassical ones (dashed lines) obtaned via the TETF functionals in terms of the exact densities $\rho(\boldsymbol{r})$. 

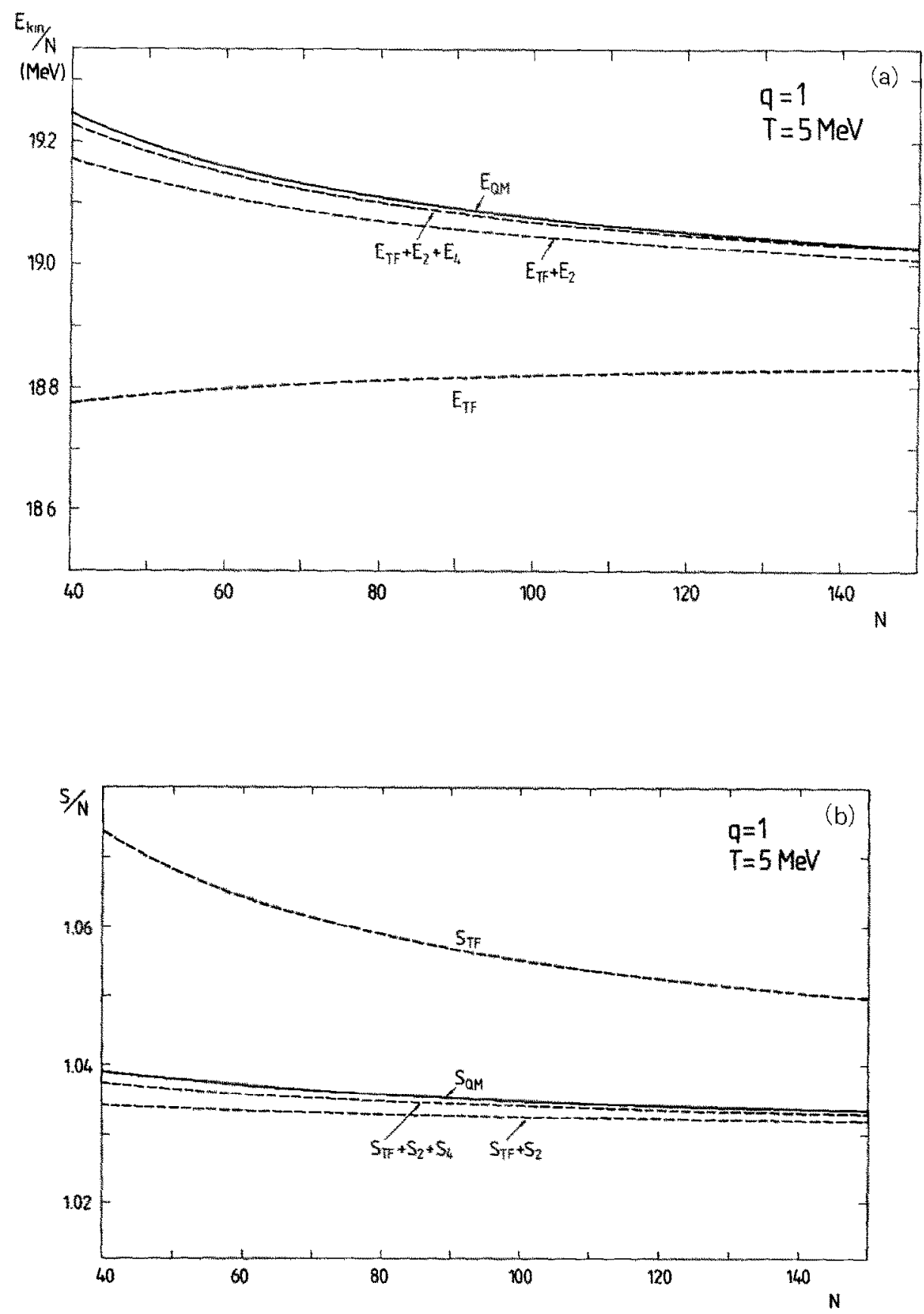

Fig. 3. Kinetic energy $E_{\text {kxis }}$ (a) and entropy $S(b)$ versus particlc number $N$ for $T=5 \mathrm{MeV}$. The contributions to $E_{\mathrm{km}}$ and $S$ of the different orders of the functionals $(3.8)$ and (3.13) are shown (dashed lines) and the result with the full TETF functionals is compared wh the quantum-medhanical result (full line). 
look puzzling that at temperatures as high as $T=5 \mathrm{MeV}$ quantum oscillations still persist, whereas it was shown above that all shell effects have been washed out for the integrated quantities at these temperatures. This behaviour is quite similar to the one observed with the Strutinsky-averaging procedure where one needs a parameter $\gamma$ about twice as large to smooth out the oscillations of density profiles than the one needed for smoothing the single-particle energies ${ }^{37}$ ). Similarly as for the kinetic energy density in eq. (4.13), one might try to find an equivalent entropy density $\bar{\sigma}(\boldsymbol{r})$ by adding any divergence of a vector field that vanishes at infinity. It would give identical results for the entropy $S$, but might be smooth in $r$-space. In this sense the quantum oscillations of $\sigma(\boldsymbol{r})$ defined by eq. (2.22) have a certain arbitrariness and should not be given too much physical significance.

Looking at integrated quantities we give in fig. 3 the total kinetic energy $E_{\mathrm{knn}}$ and the entropy $S$ as functions of the particle number $N$. For a temperature $T=5 \mathrm{MeV}$

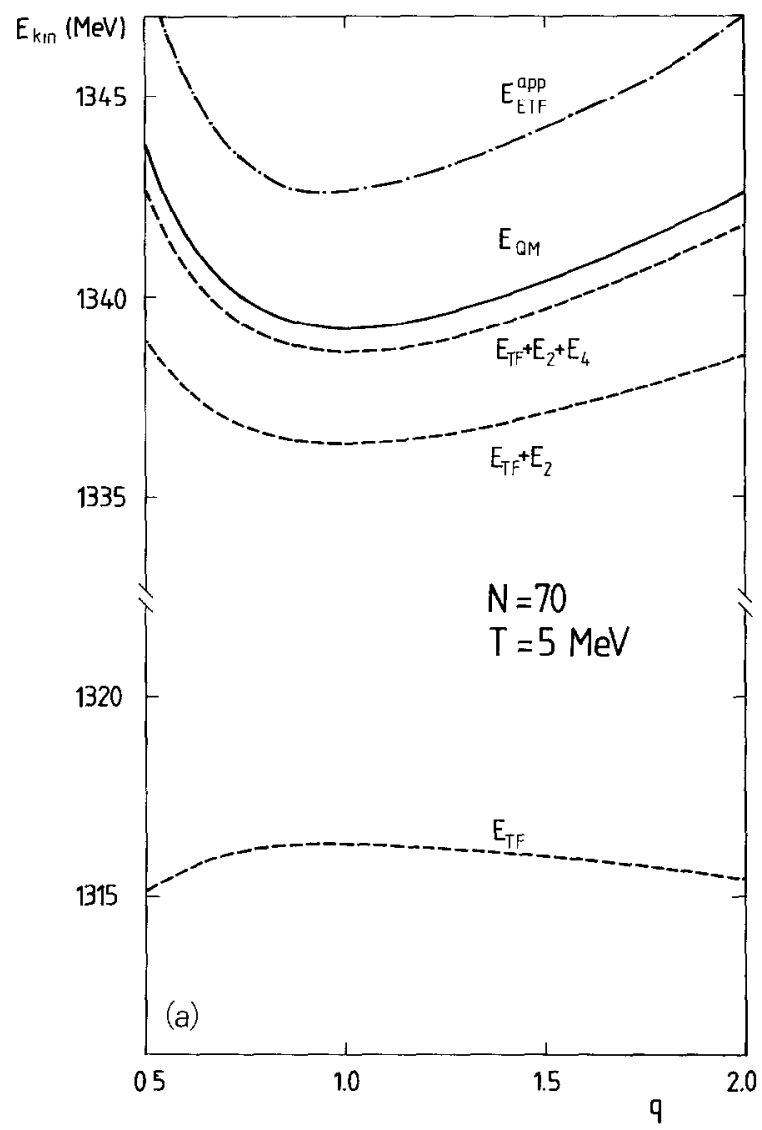

Fig. 4. Same as fig. 3, but for fixed particle number $N=70$ as a function of the deformation parameter $q$. The top most curve is the result obtained with the approximate functional, eq. (5.39). 


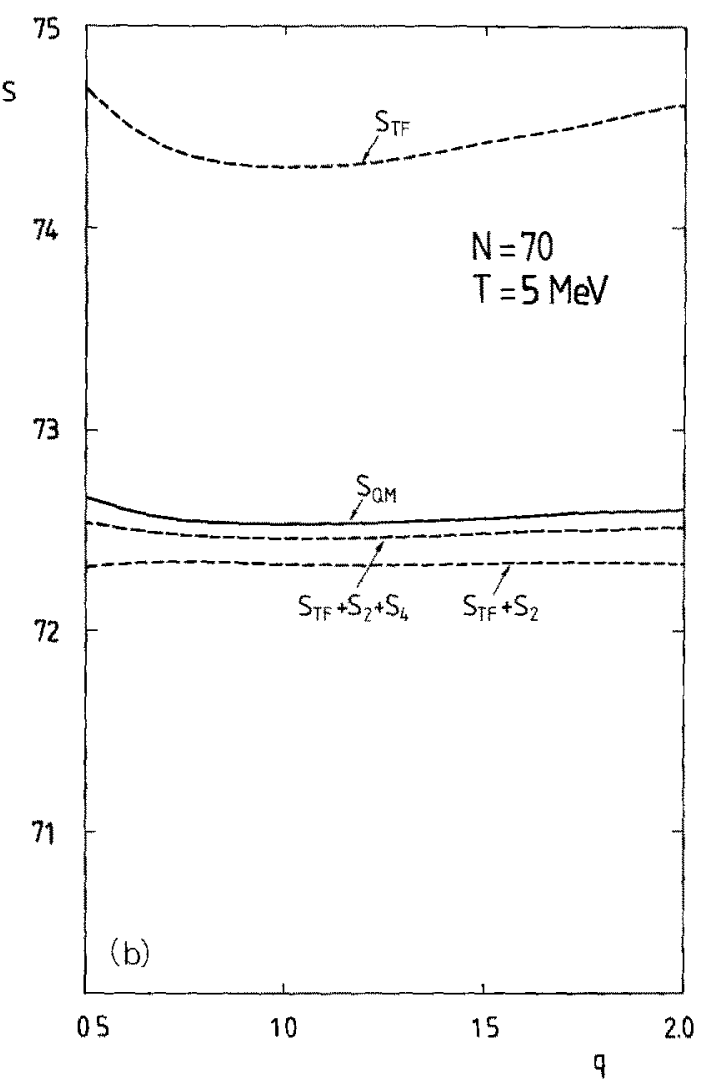

Fig. 4. (continued).

the contributions at each level of the semiclassical approximation (TF, second and fourth order) are shown. The exact $E_{\text {kin }}$ and $S$ were obtained by integrating $\tau(\boldsymbol{r})$, eq. (4.4), and $\sigma(\boldsymbol{r})$, eq. (2.22). (Note the scales used!) It turns out that going to fourth order in the functionals the exact entropy is reproduced to within less than $1 \%$ for all particle numbers shown. This agreement is still better for the kinetic energy where the deviation is less than $1 \%$ and even $0.1 \%$ for $N \geqslant 140$. As already seen from fig. 1 , shell effects no longer play any role at the temperature of $T=5$ $\mathrm{MeV}$ chosen here.

To demonstrate that the functionals derived are valid not only for spherical bui also for deformed shapes we show in fig. 4 the kinetic energy and entropy for $N=70$ particles as a function of the deformation parameter $q$. The complete functionals up to fourth order are seen to give the correct deformation dependence - which is for instance not at all the case at the TF level as already observed for $T=0$ in ref. ${ }^{16}$ ) - and deviate from the exact results by less than 1 to 2 parts in thousand. This small deviation is to be explained by higher-order corrections which obviously contribute very little. Due to the smallness of the fourth-order correction to the 
TABLE 2

Relative contributions to free energy and entropy of $N=70$ particles as in table 1, but obtained this time through the various gradient correction terms of the functionals $\mathscr{F}_{\mathrm{TETF}}[\rho]$ and $\sigma_{\mathrm{TETF}}[\rho]$

\begin{tabular}{ccccc}
\hline $\begin{array}{c}T \\
{[\mathrm{MeV}]}\end{array}$ & $F_{2} / F_{\text {TETF }}$ & $F_{1} / F_{\text {TETF }}$ & $S_{2} / S_{\text {TETF }}$ & $S_{1} / S_{\text {TETF }}$ \\
\hline 1 & & 0.004 & 0.255 & 0.069 \\
2 & 0.015 & 0.003 & 0.113 & 0.015 \\
3 & 0.015 & 0.003 & 0.065 & 0.006 \\
4 & 0.015 & 0.002 & 0.041 & 0.003 \\
5 & 0.015 & 0.002 & 0.027 & 0.002 \\
\hline
\end{tabular}

entropy it even seems reasonable to limit oneself to second order, $S_{\mathrm{TF}}+S_{2}$. For the total kinetic energy, however, it appears important to correctly include the term $\tau_{4}[\rho]$ into the calculation. Replacing the finite- $T$ functional $\tau_{4}[\rho]$ by its form valid at $T=0$, a total kinetic energy $E_{\mathrm{E} T \mathrm{ap}}^{\mathrm{ap}}$ is obtained which overestimates the exact $E_{\text {kun }}$ by $\sim 4 \mathrm{MeV}$ and gives an energy which is no better than if the fourth-order correction were omitted altogether. To study the importance of the different gradient corrections, we show their relative contributions to the total free energy and the entropy in table 2. They are seen to decrease with increasing temperatures (except for $F_{2}$, the contribution of which stays approximately constant).

\section{Density variational calculations}

In this section we shall discuss the Euler differential equation which follows from a density variational calculation with the TETF functionals developed above. We shall limit ourselves here to a one-component nuclear system (i.e. with only one kind of nucleons) without Coulomb interaction. We do not discuss here isolated, metastable nuclei at finite temperature; they will be the object of a forthcoming publication. Instead, we consider a nucleus consisting of $N$ neutrons in thermodynamical equilibrium with a surrounding neutron gas. In order to make the calculation selfconsistent, we use the energy density obtained in the Hartree-Fock approximation for a Skyrme-type effective nucleon-nucleon interaction ${ }^{28}$ ), with variable density dependence ${ }^{31}$ ):

$$
\begin{aligned}
\mathscr{E}_{\text {sky }}(\boldsymbol{r})= & \frac{\hbar^{2}}{2 m^{*}(\boldsymbol{r})} \tau(\boldsymbol{r})+\frac{3}{8} t_{0} \rho^{2}(\boldsymbol{r})+\frac{1}{16} t_{3} \rho^{2+\alpha}(\boldsymbol{r}) \\
& +b[\nabla \rho(\boldsymbol{r})]^{2}+\boldsymbol{W}(\boldsymbol{r}) \cdot \boldsymbol{J}(\boldsymbol{r}) .
\end{aligned}
$$

$J(r)$ is the spin-orbit density, eq. (2.47). The effective mass $m^{*}(r)$ and the spin-orbit 
potential $\boldsymbol{W}(\boldsymbol{r})$ are $^{28}$ )

$$
\begin{aligned}
& \frac{m}{m^{*}(r)}=f(\boldsymbol{r})=1+\beta \rho(\boldsymbol{r}) \\
& \boldsymbol{W}(\boldsymbol{r})=\frac{3}{4} W_{0} \nabla \rho(\boldsymbol{r})
\end{aligned}
$$

the coefficients $b$ and $\beta$ are given in terms of the Skyrme parameters by

$$
\begin{aligned}
& b=\frac{1}{64}\left[9 t_{1}-5 t_{2}\left(1+\frac{4}{5} x_{2}\right)\right], \\
& \beta=\frac{2 m}{\hbar^{2}} \frac{1}{16}\left[3 t_{1}+5 t_{2}\left(1+\frac{4}{5} x_{2}\right)\right] .
\end{aligned}
$$

In principle, we have to question to what extent the Skyrme parameters $t_{0}, t_{1}, t_{2}$, $x_{2}, t_{3}, \alpha$ and $W_{0}$ will themselves depend on the temperature $T$. This could theoretically be decided by a finite-temperature Brueckner calculation and a density matrix expansion such as performed by Negele and Vautherin in the $T=0$ case $^{32}$ ). As long as such calculations are not available, we make the same assumption as in all Skyrme calculations at $T>0$ done so far $^{5-10}$ ), i.e. keeping the Skyrme parameters determined by nuclear ground-state properties.

The energy which is made stationary at $T>0$ is the Gibbs free energy:

$$
\delta \int \mathrm{d}^{3} r\left\{\mathscr{E}_{\text {sky }}(\boldsymbol{r})-T \boldsymbol{\sigma}(\boldsymbol{r})-\lambda \rho(\boldsymbol{r})+P_{0}\right\}=0,
$$

where the chemical potential $\lambda$ plays the rôle of a Lagrange multiplier fixing the nucleon number, and $P_{0}$ is the external pressure needed to maintain the thermodynamical equilibrium. Using the TETF functional developed in sect. 3 for the free energy density, we can rewrite eq. (5.6) as a variational equation for the density $\rho(r)$ :

$$
\frac{\delta}{\delta \rho(\boldsymbol{r})} \int \mathrm{d}^{3} r\left\{\mathscr{F}_{\text {TETF }}[\rho]-\lambda \rho+P_{0}\right\}=0 \text {. }
$$

$\mathscr{F}_{\text {TETF }}[\rho]$ is now given by eqs. (3.8)-(3.10) and (3.19) after replacing the potential term $V \rho$ in $\mathscr{F}_{T F}[\rho]$, eq. (3.9), by the right-hand side of eq. (5.1) excepting the first term. It is useful to split $\mathscr{F}_{\text {ETF }}[\rho]$ into a homogeneous term $\mathscr{F}_{\infty}(\rho)$, which is the free energy density of symmetric infinite nuclear matter, and the sum of all gradient terms. Using eqs. (2.48) and (5.3) in the spin-orbit energy, we obtain

$$
\mathscr{F}_{\text {TETF }}[\rho]=\mathscr{F}_{\infty}(\rho)+\left[b-\left(\frac{3}{4} W_{0}\right)^{2} \frac{2 m}{\hbar^{2}} \frac{1}{f} \rho\right](\nabla \rho)^{2}+\mathscr{F}_{2}[\rho]+\mathscr{F}_{4}[\rho]
$$

with

$$
\mathscr{F}_{\infty}(\rho)=-\frac{2}{3} T A_{T}^{*} J_{3 / 2}(\eta)+T \eta \rho+\frac{3}{8} t_{0} \rho^{2}+\frac{1}{16} t_{3} \rho^{2+\alpha} .
$$

Note that the TF part of the term proportional to $\tau \rho$ of the Skyrme energy is included in the first term of eq. (5.9). The second- and fourth-order terms $\mathscr{F}_{2}[\rho]$ and 
$\mathscr{F}_{4}[\rho]$ are given by eqs. (3.10) and (3.19), respectively. Since $\mathscr{F}_{2}[\rho]$ is proportional to $(\nabla \rho)^{2}$, it further simplifies the notation to collect all coefficients of $(\nabla \rho)^{2}$ in eqs. (3.10) and (5.8) into one function $s(\rho)$ and to finally write

$$
\mathscr{F}_{\text {TETF }}[\rho]=\mathscr{F}_{\infty}(\rho)+s(\rho)(\nabla \rho)^{2}+\mathscr{F}_{4}[\rho] .
$$

Performing now the variation in eq. (5.7), we obtain the Euler equation

$$
\mathscr{F}_{\infty}^{\prime}(\rho)-2 s(\rho) \Delta \rho-s^{\prime}(\rho)(\nabla \rho)^{2}+\frac{\delta \mathscr{F}_{4}[\rho]}{\delta \rho}=\lambda .
$$

The primes on functions of $\rho$ shall henceforth denote derivatives with respect to $\rho$. The functional derivative $\delta \mathscr{F}_{4}[\rho] / \delta \rho$ contains eight terms with up to fourth-order gradients of $\rho$ with complicated coefficients. This makes eq. (5.11) a highly nonlinear fourth-order partial differential equation which in general will be very difficult to solve even in the spherical case.

One practical way out of this difficulty is to parametrize the density profile $\rho(\boldsymbol{r})$ and to minimize the total free energy with respect to the parameters. This procedure has been followed in the $T=0$ case and led to an excellent agreement with Strutinsky-averaged Hartree-Fock results ${ }^{10}$ ).

The second possibility, chosen here, is to perform a "leptodermous", i.e. liquiddrop model (LDM) type expansion ${ }^{10,33}$ ) of the free energy:

$$
F=a_{\mathrm{v}} A+a_{\mathrm{s}} A^{2 / 3}+a_{\mathrm{c}} A^{1 / 3}+\cdots,
$$

where $a_{\mathrm{v}}$ is the volume (free) energy constant. Under the assumption that the density of the nucleus is essentially flat in the interior and varies only in a relatively narrow surface region, the LDM parameters $a_{\mathrm{s}}, a_{\mathrm{c}}$ etc. can be systematically gained from a semi-infinite nuclear matter calculation. Strictly speaking, the surface tension and the Coulomb repulsion tend to spoil this leptodermous behaviour, the former leading to an increased central density in light nuclei and the latter to a central depression of $\rho(\boldsymbol{r})$ in heavy nuclei. These modifications of the density profile have, however, heen shown in realistic calculations ${ }^{10}$ ) to affect the total hinding energies by less than $\sim 1 \mathrm{MeV}$, so that the leptodermous assumption is well justified in calculations of total energies for nuclei with $A \geq 40$.

We thus apply the density variational principle to the surface free energy given by

$$
a_{\mathrm{s}}=4 \pi r_{0}^{2} \sigma=4 \pi r_{0}^{2} \int_{-\infty}^{\infty} \mathrm{d} z\left\{\mathscr{F}_{\mathrm{TETF}}[\rho(z)]-\lambda \rho(z)+P_{0}\right\},
$$

where $\rho(z)$ is the one-dimensional profile of semi-infinite nuclear matter. At finite temperature, $a_{s}$ is the interface energy defined by Ravenhall et al. ${ }^{21}$ ) of the profile connecting the condensed (i.e. liquid) nuclear matter with density $\rho_{0}$ and the nuclear gas with density $\rho_{\mathrm{g}}$. Thus the density profile $\rho(z)$ has the limits

$$
\rho(z) \underset{z \rightarrow-\infty}{\longrightarrow} \rho_{0}, \quad \rho(z) \underset{z \rightarrow+\infty}{\longrightarrow} \rho_{\mathrm{g}} .
$$

For a given temperature $T>0$, the four quantities $\lambda, P_{0}, \rho_{0}$ and $\rho_{\mathrm{g}}$ are determined 
by the Maxwell construction which consists in solving simultaneously the two sets of equations

$$
\begin{aligned}
\lambda & =\mathscr{F}_{\infty}^{\prime}\left(\rho_{0}\right)=\mathscr{F}_{\infty}^{\prime}\left(\rho_{\mathrm{g}}\right), \\
P_{0} & =\lambda \rho_{0}-\mathscr{F}_{\infty}\left(\rho_{0}\right)=\lambda \rho_{\mathrm{g}}-\mathscr{F}_{\infty}\left(\rho_{\mathrm{g}}\right) .
\end{aligned}
$$

At zero temperature where $P_{0}=0$, eq. (5.13) reduces to the standard expression of the surface energy ${ }^{33}$ ); in this case the chemical potential $\lambda$ is identical with the binding energy per nucleon of infinite nuclear matter-

$$
\lambda=\mathscr{E}_{\infty}^{\prime}\left(\rho_{0}\right)=\frac{\mathscr{E}_{\infty}\left(\rho_{0}\right)}{\rho_{0}}=a_{\mathrm{v}}, \quad(T=0) .
$$

The nuclear radius constant $r_{0}$ in eq. (5.13) is given by

$$
r_{0}=\left(\frac{4}{3} \pi \rho_{0}\right)^{-1 / 3} \text {. }
$$

With this one-dimensional geometry, the Euler equation (5.11) can be simplified, because the variable $z$ does not appear explicitly. Noting that $\mathscr{F}_{4}[\rho]$ is now of the form

$$
\mathscr{F}_{4}[\rho]=g(\rho)\left(\rho^{\prime}\right)^{4}+h(\rho)\left(\rho^{\prime \prime}\right)^{2}+l(\rho)\left(\rho^{\prime}\right)^{2} \rho^{\prime \prime},
$$

where the primes on $\rho$ denote derivatives with respect to $z$, the Euler equation (5.11) becomes

$$
\begin{aligned}
\mathscr{F}_{\infty}^{\prime}(\rho)-2 s(\rho) \rho^{\prime \prime}-s^{\prime}(\rho)\left(\rho^{\prime}\right)^{2}-12 g(\rho)\left(\rho^{\prime}\right)^{2} \rho^{\prime \prime}-3 g^{\prime}(\rho)\left(\rho^{\prime}\right)^{4} \\
+2 h(\rho) \rho^{(4)}+h^{\prime}(\rho)\left[4 \rho^{\prime} \rho^{\prime \prime \prime}+3\left(\rho^{\prime \prime}\right)^{2}\right]+2 h^{\prime \prime}(\rho)\left(\rho^{\prime}\right)^{2} \rho^{\prime \prime} \\
+4 l^{\prime}(\rho)\left(\rho^{\prime}\right)^{2} \rho^{\prime \prime}+l^{\prime \prime}(\rho)\left(\rho^{\prime}\right)^{4}=\lambda .
\end{aligned}
$$

This is still a rather unpleasant nonlinear fourth-order differential equation. (The reader will not confuse the derivatives $s^{\prime}(\rho), l^{\prime \prime}(\rho)$, etc. with respect to $\rho$ and those with respect to $\left.z: \rho^{\prime}, \rho^{\prime \prime} !\right)$

The nice feature now is that eq. (5.20) can be integrated once after the substitution

$$
p=\left[\rho^{\prime}(z)\right]^{2}=p(\rho) \text {. }
$$

Expressing all spatial derivatives of $\rho$ via eq. (5.21) through $p(\rho)$ and its derivatives with respect to $\rho$, one finds that the left-hand side of eq. (5.20) can be written as a total differential. The equation can thus be integrated once over $\rho$, whereby the integration constant is the pressure $P_{0}$, so that one is left with the following second-order differential equation for $p(\rho)$ :

$$
s p+\left(3 g-l^{\prime}\right) p^{2}+\frac{1}{4} h\left(p^{\prime}\right)^{2}-h^{\prime} p p^{\prime}-h p p^{\prime \prime}=\Omega ;
$$

here we have introduced the energy density

$$
\Omega(\rho)=\mathscr{F}_{\infty}(\rho)-\lambda \rho+P_{0}
$$

whose spatial integral is the thermodynamical (or grand canonical) potential. 
The boundary conditions for $p(\rho)$ are very simple. In the two limits $z \rightarrow \pm \infty$, all spatial derivatives of $\rho(z)$ vanish, and thus also $p$, and eq. (5.20) reduces to the first set of equilibrium conditions, eq. (5.15). On the other hand, eq. (5.16) implies that also $\Omega(\rho)$, eq. (5.23), vanishes at the boundaries. Looking at eq. (5.22) we see that also $p^{\prime}(\rho)$ must be zero there, so that the boundary conditions for $p(\rho)$ are

$$
p\left(\rho_{0}\right)=p\left(\rho_{\mathrm{g}}\right)=p^{\prime}\left(\rho_{0}\right)=p^{\prime}\left(\rho_{\mathrm{g}}\right)=0 .
$$

From eq. (5.21) it is furthermore obvious that $p(\rho)$ must be positive definite in between the boundaries.

Once eq. (5.22) for $p(\rho)$ has been solved, one can obtain the inverse profile function $z(\rho)$ from eq. (5.21) by a simple quadrature:

$$
z(\rho)=-\int_{\rho_{\mathrm{g}}}^{\rho} \frac{1}{\sqrt{p\left(\rho^{\prime}\right)}} \mathrm{d} \rho^{\prime}+C .
$$

The integration constant $C$, which is formally infinite with the choice of eq. (5.14), is practically irrelevant since the surface energy, eq. (5.13), is invariant under a coordinate translation along $z$. Using eqs. (5.21) and (5.22), the surface tension $\sigma$, eq. (5.13), can, in fact, be expressed directly as an integral over $\rho$ which after some partial integrations takes the form

$$
\sigma=\int_{\rho_{\mathrm{g}}}^{\rho_{0}} \frac{\mathrm{d} \rho}{\sqrt{p(\rho)}}\left[\frac{4}{3} \Omega(\rho)+\frac{2}{3} s(\rho) p(\rho)\right] .
$$

This result can also be obtained directly from eq. (5.13) requiring that $a_{\mathrm{s}}$ should be stationary with respect to a scale transformation $z \rightarrow z^{\prime}=z / \alpha$, just as one derives virial theorems for bound systems. With eq. (5.10) this leads to

$$
\sigma=\int_{-\infty}^{\infty} \mathrm{d} z\left\{\frac{4}{3}\left[\mathscr{F}_{\infty}(\rho)-\lambda \rho+P_{0}\right]+\frac{2}{3} s(\rho)\left(\rho^{\prime}\right)^{2}\right\},
$$

which after the substitution (5.21) reduces to eq. (5.26).

The coefficient of the term proportional to $A^{1 / 3}$ in the total free energy eq. (5.12) can be written as

$$
a_{\mathrm{c}}=8 \pi r_{0} \kappa+a_{\text {comp }} .
$$

The first term is the proper curvature energy with $\kappa$ given in this case by ${ }^{10}$ )

$$
\begin{aligned}
\kappa= & \int_{-\infty}^{\infty}\left(z-z_{0}\right)\left\{\mathscr{F}_{\mathrm{TETF}}[\rho]-\lambda \rho+P_{0}\right\} \mathrm{d} z \\
& +\int_{-\infty}^{\infty} \rho^{\prime}\left[l\left(\rho^{\prime}\right)^{2}+2 h \rho^{\prime \prime}\right] \mathrm{d} z,
\end{aligned}
$$

where

$$
z_{0}=\frac{1}{\left(\rho_{\mathrm{g}}-\rho_{0}\right)} \int_{-\infty}^{\infty} z \rho^{\prime}(z) \mathrm{d} z=\frac{1}{\left(\rho_{0}-\rho_{\mathrm{g}}\right)} \int_{\rho_{\mathrm{g}}}^{\rho_{0}} z(\rho) \mathrm{d} \rho .
$$


With eqs. (5.21) and (5.22), $\kappa$ can be re-expressed in the form

$$
\begin{aligned}
\kappa=\int_{\rho_{\mathrm{g}}}^{\rho_{0}}\left\{\frac{1}{\sqrt{p(\rho)}}\left[z(\rho)-z_{0}\right]\left[\frac{4}{3} \Omega(\rho)+\frac{2}{3} s(\rho) p(\rho)\right]\right. \\
\left.+\frac{2}{3}\left[l(\rho) p(\rho)+h(\rho) p^{\prime}(\rho)\right]\right\} \mathrm{d} \rho,
\end{aligned}
$$

which is easily seen to be independent of the integration constant $C$ in eq. (5.25). The second term in eq. (5.28) is the so-called compression energy ${ }^{33}$ ) stemming from the fact that the central density $\tilde{\rho}_{0}$ in a finite nucleus is slightly increased with respect to the infinite matter density $\rho_{0}$ due to the surface tension and the finite compressibility of nuclear matter. To lowest order in the droplet model expansion of the total energy in powers of the small quantity $\tilde{\rho}_{0}-\rho_{0}$ one finds ${ }^{33}$ )

$$
a_{\text {comp }}=-2 a_{\mathrm{s}}^{2} / K_{\infty}
$$

where $K_{\infty}$ is the incompressibility of the condensed infinite matter phase given at $T \geqslant 0$ by

$$
K_{\infty}=9 \rho_{0} \mathscr{F}_{\infty}^{\prime \prime}\left(\rho_{0}\right)
$$

The contribution $a_{\text {comp }}$ is usually small, of the order of $\sim 10-20 \%$ of the total $a_{c}$, for all realistic interactions ${ }^{10}$ ).

The nonlinear second-order differential equation (5.22) for $p(\rho)$ with the boundary conditions (5.24) is most easily solved by decomposing it into a system of two first-order equations by the substitution

$$
q(\rho)=h(\rho) p(\rho) p^{\prime}(\rho)
$$

One then has to solve the coupled equations

$$
\left.\begin{array}{c}
p^{\prime}=\frac{q}{h p} \\
q^{\prime}=s p+\left(3 g-l^{\prime}\right) p^{2}+\frac{5}{4} \frac{q^{2}}{h p^{2}}-\Omega
\end{array}\right\}
$$

The division by $p$ and $h$ causes no difficulty, since both functions are positive definite for $\rho_{\mathrm{g}}<\rho<\rho_{0}$. At the boundaries, we have $q=q^{\prime}=0$.

We have solved eqs. (5.35) numerically using a finite-difference technique ${ }^{34}$ ) with Newton iteration, starting from approximate solutions $p_{0}(\rho)$ and $q_{0}(\rho)$. As starting approximation we took a parametrized density profile of the form

$$
\rho(z)=\rho_{\mathrm{g}}+\frac{\rho_{0}-\rho_{\mathrm{g}}}{\left(1+\mathrm{e}^{z / \alpha}\right)^{\gamma}},
$$




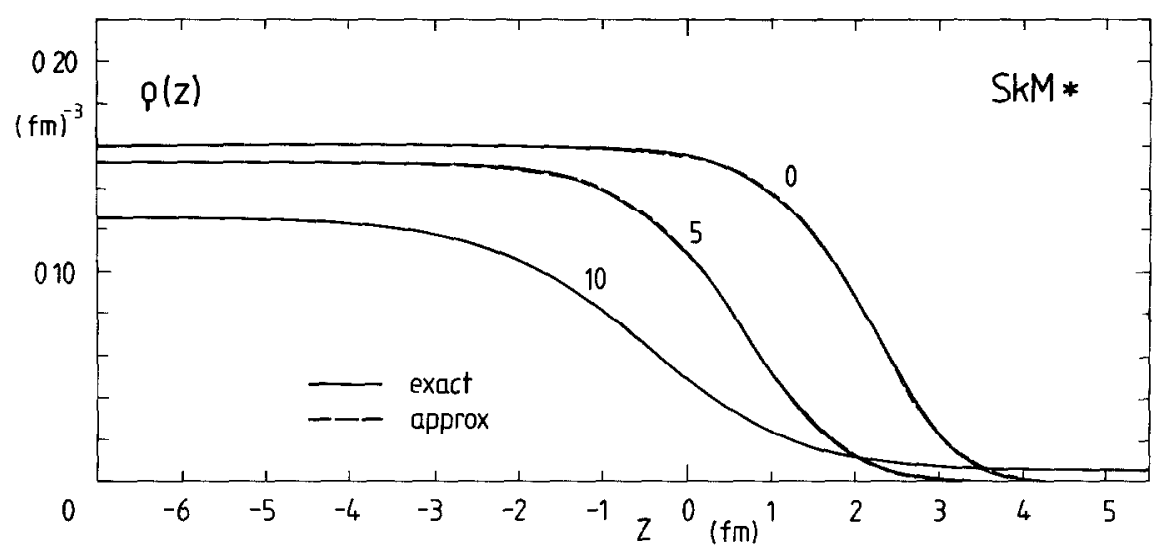

Fig. 5. Profiles of the interface between condensed (left) and gaseous (right) symmetric nuclear matter at various temperatures $T$ (given in MeV). Solid lines: exact numerical solutions of the Euler equation (5.22).

Dashed lines: variational densities parametrized as in eq. (5.36). The force $\mathrm{SkM}^{*}$ was used ${ }^{36}$ ).

which was used in earlier variational calculations ${ }^{10,35}$ ). It leads to

$$
p_{0}(\rho)=\left(\frac{\gamma}{\alpha}\right)^{2}\left(\rho-\rho_{\mathrm{g}}\right)^{2}\left[1-\left(\frac{\rho-\rho_{\mathrm{g}}}{\rho_{0}-\rho_{\mathrm{g}}}\right)^{1 / \gamma}\right]^{2}
$$

In order to be consistent with the earlier calculations ${ }^{10,35}$ ), we included in the coefficients $g(\rho), h(\rho)$ and $l(\rho)$ also all those spin-orbit and effective mass contributions to $\mathscr{F}_{4}[\rho]$, left out in the derivation in subsect. 3.2 , in their form valid for $T=0$. These terms, which are found in their simplest form in ref. ${ }^{10}$ ), have only a minor influence on the results and therefore the neglect of their temperature dependence should not be serious ${ }^{\dagger}$.

In fig. 5 we show the density profiles obtained at various temperatures with the Skyrme force SkM* which gives excellent ground-state properties of stable spherical nuclei ${ }^{36}$ ). The solid lines are the exact numerical solutions of eqs. (5.35) and (5.25), whereas the dashed lines show the profiles, eq. (5.36) with the variational parameters $\alpha$ and $\gamma$ which minimize $a_{\text {s }}$, eq. (5.13), at each temperature. The small differences demonstrate that a restricted variational calculation with trial densities of the form (5.36) gives already very good solutions.

In fig. 6 we present the LDM parameters $a_{\mathrm{s}}$ and $a_{\mathrm{c}}$, evaluated according to eqs. (5.13), (5.26)-(5.33) versus the temperature $T$. The solid lines are the exact results obtained with the numerical solution of the full Euler equation (5.22). The dasheddotted lines are obtained if the fourth order term $\mathscr{F}_{4}[\rho]$ is omitted (i.e. putting

\footnotetext{
To leading order, it is sufficient to replace the nucleon mass $m$ by $m^{*}(\boldsymbol{r})$ everywhere in $\mathscr{F}_{4}[\rho]$.
} 


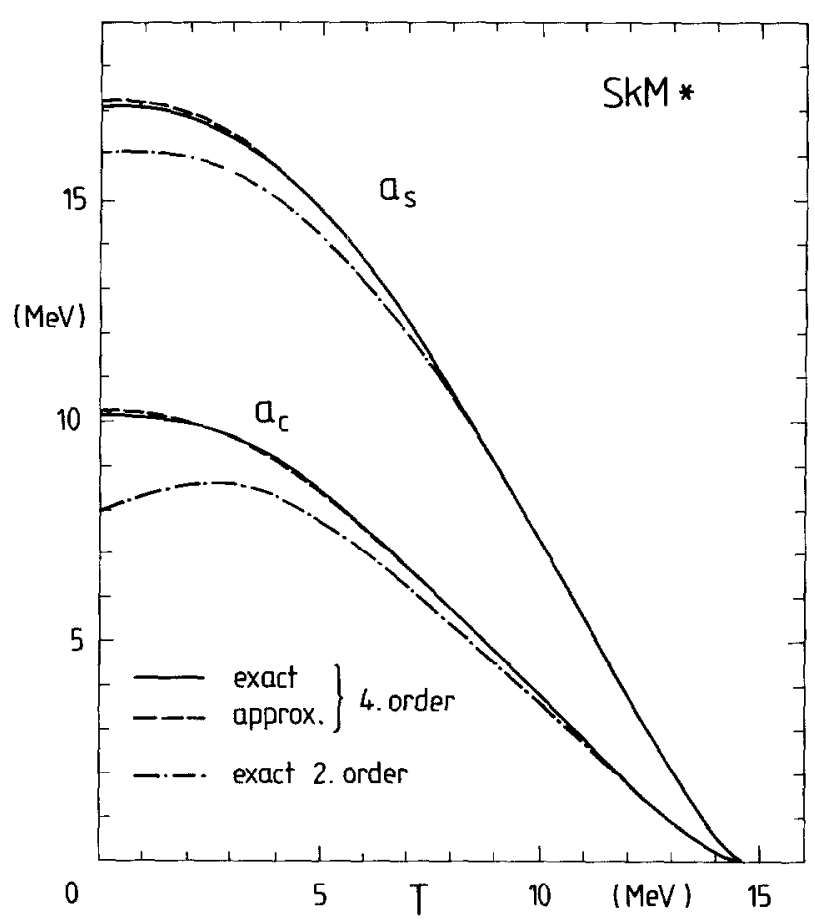

Fig. 6. Surface energy $a_{\mathrm{s}}$ and curvature energy $a_{\mathrm{c}}$ (including the compression energy) versus $T$, obtanned with the SkM* force. Solid lines: using exact solution of the full Euler equation. Dashed lines: variational result with the parametrized density profiles (5.36). Dashed-dotted lines: using exact solution of Euler equation truncated after the second-order gradient terms.

$g(\rho)=g(\rho)=l(\rho)=0) ;$ the solution for $p(\rho)$ is then simply

$$
p(\rho)=\Omega(\rho) / s(\rho) .
$$

The dashed lines finally correspond to the results obtained with the full functional $\mathscr{F}_{\text {TETF }}[\rho]$, but using the parametrized density profiles, eq. (5.36), and minimizing $a_{s}$ with respect to $\alpha$ and $\gamma$.

Both parameters $a_{\mathrm{s}}$ and $a_{\mathrm{c}}$ go to zero at the critical temperature $T_{\text {crit }}=14.6 \mathrm{MeV}$ where the liquid and gas densities $\rho_{0}$ and $\rho_{\mathrm{g}}$ become equal ${ }^{10}$ ). Beyond $T_{\text {crt }}$, only one phase exists and the parameters $a_{\mathrm{s}}, a_{\mathrm{c}}$ lose their meaning.

The results obtained with the parametrized trial densities reproduce the exact solutions within less than $1 \%$ at low temperatures and within less than $0.1 \%$ for $T \gtrsim 5 \mathrm{MeV}$. This gives a very nice a posteriori justification of the restricted variational approach used earlier ${ }^{10,35}$ ). We also learn from fig. 6 that the fourth-order gradient corrections become less important at higher temperatures, as already noticed in sect. 4. For $0 \leq T \leq 3 \mathrm{MeV}$, however, they are clearly necessary to obtain reliable LDM parameters, a conclusion which was already drawn from calculations 
at $T=0\left[\right.$ ref. $\left.\left.{ }^{10}\right)\right]$. In the earlier calculations, we had used an approximate TETF functional $\mathscr{F} \mathscr{T}_{\mathrm{TETF}}[\rho]$ in which the fourth-order term was taken in its form valid at $T=0$, i.e.

$$
\mathscr{F}_{\mathrm{TETF}}^{\mathrm{app}}[\rho]=\mathscr{F}_{\mathrm{TF}}^{T>0}[\rho]+\mathscr{F}_{2}^{T>0}[\rho]+\frac{\hbar^{2}}{2 m} f_{4}^{T=0}[\rho] ;
$$

hereby the $\sigma_{4}[\rho]$ contribution to the entropy was neglected. The LDM parameters obtained with this approximation ${ }^{10,35}$ ) are practically the same as those which we obtained here with the full functional $\mathscr{F}_{\mathrm{TETF}}[\rho]$ (shown by the dashed lines in fig. 6 ); the surface energies $a_{\mathrm{s}}$ agree within less than $0.5 \%$ and the parameters $a_{\mathrm{c}}$ within less than $1.5 \%$. This accords with our model results of sect. 4 : The contribution from $\sigma_{4}[\rho]$ to the total entropy is always very small; the temperature-dependent part of $\tau_{4}[\rho]$ contributes up to several $\mathrm{MeV}$ in the total kinetic energy for finite nuclei and thus is not negligible, but it shows up very little in the LDM parameters $a_{\mathrm{s}}$ and $a_{\mathrm{c}}$ which must be multiplied by $A^{2 / 3}$ and $A^{1 / 3}$, respectively, to give their contributions to the total energy. In table 3 we present the surface energies $a_{\mathrm{s}}$ obtained for $T=0$ for a series of current Skyrme force parametrizations, obtained both from the exact solution of the Euler equation and with the trial density profiles, eq. (5.36). The difference is roughly the same for all forces and smaller than $1 \%$.

The asymmetric - or isovectorial - LDM parameters can be obtained along the same lines, treating neutrons and protons separately; in this case one has to solve two coupled Euler equations. Their systematical evaluation will be the object of a future publication.

Surface energy coefficients $a_{s}$ for semi-infinite nuclear matter at $T=0$ have also been calculated in the Hartree-Fock (HF) approximation using various Skyrme forces ${ }^{45}$ ). The agreement with the semiclassical results for $a_{\mathrm{s}}$ is very good (within $\leq 4 \%$ ), in particular with respect to the variation of $a_{s}$ with the parameters of the force. As already discussed earlier ${ }^{10,46}$ ), the HF results are typically higher than the ETF values by $0.5-0.8 \mathrm{MeV}$. A smaller part of the difference $(\sim 0.1-0.3 \mathrm{MeV})$ is

TABLE 3

Surface energy coefficient $a_{\text {s }}$ obtained from the semi-infinite nuclear matter profiles at $T=0$ with various Skyrme forces

\begin{tabular}{cccc}
\hline Force & $a_{\mathrm{s}}^{\text {ex }}[\mathrm{MeV}]$ & $a_{\mathrm{s}}^{\text {par }}$ & $\Delta a$ \\
\hline $\mathrm{SIII}^{\mathrm{a}}$ ) & 17.91 & 18.04 & 0.13 \\
$\mathrm{Ska}^{\mathrm{b}}$ ) & 18.37 & 18.51 & 0.14 \\
$\mathrm{SkM}^{\mathrm{c}}$ ) & 16.45 & 16.60 & 0.15 \\
$\left.\mathrm{SkM}^{* \mathrm{~d}}\right)$ & 17.07 & 17.22 & 0.15 \\
To83 $\left.^{\mathrm{e}}\right)$ & 17.27 & 17.43 & 0.16 \\
\hline
\end{tabular}

The first column (ex) gives the results obtained from the exact numerical solution of the Euler equation. The second column (par) shows the variational results using the parametrized density profiles, (5.36). $\Delta a$ is the difference. The numerical uncertainty of $a_{s}^{\mathrm{ex}}$ is $\pm 0.03 \mathrm{MeV}$, that of $a_{\mathrm{s}}^{\text {par }}$ less than $\pm 0.01 \mathrm{MeV}$.
$\left.{ }^{a}\right)$ Ref. ${ }^{38}$ ).
$\left.{ }^{b}\right)$ Ref. ${ }^{39}$ ).
c) Ref. ${ }^{40}$ ).
d) $\operatorname{Ref}^{36}$ ).
e) Ref. ${ }^{41}$ ). 
related to a systematic overbinding found in the ETF variational results ${ }^{10}$ ); the largest part may be due to numerical uncertainties $(\sim 0.5 \mathrm{MeV}$ or more) of the HF results ${ }^{45}$ ). [See ref. ${ }^{46}$ ) for a systematic comparison of surface energies obtained with both models.]

\section{Summary and conclusions}

We have presented the recently developed ETF model at finite temperatures. The functionals for the free energy density $\mathscr{F}_{\text {TETF }}[\rho]$ and for the entropy density $\sigma_{\text {TETF }}[\rho]$ have been presented, for a nonlocal Skyrme-type one-body potential up to second order and for a local potential up to the fourth-order gradient corrections. For a harmonic-oscillator potential, we have shown that the Wigner-Kirkwood type $\hbar$-expansion of the free energy and the entropy converges very fast and reproduces the quantum-mechanical results very accurately for temperatures $T \geq 3 \mathrm{MeV}$ where the shell effects in the energy are washed out.

We have also demonstrated that the TETF functionals allow one to calculate the total free energy and entropy with an accuracy that reaches $0.1 \%$ or less for $T \geq 3$ $\mathrm{MeV}$, independently of the deformation of the potential and of the particle number (as long as it is not too small). This is perhaps the first time that kinetic energy and entropy of a (non-interacting!) fermion system have been calculated through the local density $\rho(\boldsymbol{r})$ alone with such a precision, and gives a very nice numerical illustration for the validity of the Hohenberg-Kohn theorem ${ }^{15,18}$ ). Since this theorem also tells us that the functionals for the uncorrelated part of the free energy, and thus also the entropy, do not depend on the special form of the potential $V(r)$ (as long as it is local), our results have a validity which is not restricted to the case of the oscillator potential chosen here as a test example.

These results encourage one to develop and use the TETF theory also for nonlocal parts of the potential, like the effective mass and spin-orbit terms whose full contributions to the functionals $\mathscr{F}[\rho]$ and $\sigma[\rho]$ have also been given here up to second order. In particular, our functionals now make it possible to perform density variational calculations for excited nuclear systems using a Skyrme-type effective interaction. In order to illustrate this, we have derived the appropriate Euler-Lagrange equation for the density of a symmetric nucleus. We have for the first time solved numerically this nonlinear, fourth-order differential equation for the case of a one-dimensional semi-infinite density profile which describes the interface (without curvature effects) of a nuclear liquid-gas two-phase system. Earlier attempts to do this in the case of simple semi-infinite nuclear matter at $T=0$ having failed, variational calculations with parametrized trial density of a modified Fermi function type have been frequently used [see ref. ${ }^{10}$ ) and the literature quoted therein]. We have shown here that, indeed, such trial densities allow to calculate the surface (free) energy from the semi-infinite profile to within $\sim 1 \%$ at $T=0$ and even more exactly at higher temperatures. 
This opens up the possibility to calculate thermal properties of excited nuclear systems in a semiclassical way which becomes quantitatively equivalent to the Hartree-Fock method for $T \geqslant 3 \mathrm{MeV}$, but which requires much less numerical effort. In particular, the problem of including correctly the continuum states ${ }^{9}$ ) is eliminated here since everything is determined by the local density $\rho(\boldsymbol{r})$. Among many interesting applications, we mention the equation of state of hot nuclear matter as used in astrophysics for the calculation of supernovae evolution, the determination of LDM parameters as functions of the temperature, or fission barriers of highly excited nuclei. Several attempts in these directions have already been undertaken ${ }^{21,23,35,42}$ ), in some cases using the TF functionals only ${ }^{21}$ ).

We want to stress here the importance of the second- and fourth-order gradient corrections for describing correctly the nuclear surface properties on which, in particular, the fission barriers depend very crucially ${ }^{10,17,23}$ ). Calculations with simplified functionals may accidentally lead to reasonable results in certain cases for certain forces, but should be taken with great care.

A remark on the so-called low-temperature expansion might also be appropriate. It is obtained if in the ETF densities (2.38)-(2.45) all functions $J_{\mu}\left(\eta_{0}\right)$ are expanded according to eq. (A.11) for large values of $\eta_{0}$ (2.41), or equivalently, if the temperature factor $\tilde{f}_{T}(\beta)$ of the Bloch density (2.18) is expanded in powers of $(\beta T)$. This leads to a simple quadratic temperature dependence of the kinetic energy density functional ${ }^{10,44}$ ). However, the limit $\eta_{0} \gg 1$ is never correct, even at very low temperatures, in the nuclear surface near and beyond the classical turning point. Therefore this approximation should not be used for finite nuclei. It has, in fact, been shown to give rather bad results ${ }^{10,35}$ ).

As we have clearly demonstrated, such simplifying assumptions are no longer necessary since the inclusion of the full functionals $\mathscr{F}_{2}[\rho]$ and $\mathscr{F}_{4}[\rho]$ with their correct temperature-dependent coefficients is quite easy and guarantees a sufficient accuracy in the kinetic (free) energy of realistic nuclear systems at arbitrary temperatures.

We have greatly benefitted from stimulating discussions with W. Stocker.

\section{Appendix A}

ANALYTICAL CONTINUATION OF THE FERMI INTEGRALS AND THEIR NUMERICAL COMPUTATION

The so-called Fermi integrals

$$
J_{\mu}(\eta)=\int_{0}^{\infty} \frac{x^{\mu}}{1+\exp (x-\eta)} \mathrm{d} x \quad(\mu>-1)
$$

are well known from the Thomas-Fermi model at finite temperature ${ }^{19-21}$ ), where the 
functions $J_{\mu}(\eta)$ occur for values $\mu=\frac{3}{2}, \frac{1}{2}$ and $-\frac{1}{2}$. In the TETF model we encounter derivatives of these functions which necessitate their analytical continuation to values of $\mu<-1$ for which the integral (A.1) is not defined. For the mathematically interested reader, we refer to a recent article by Fernández Velicia ${ }^{43}$ ) who extensively discussed these analytical continuations, their series expansions and numerical approximations. The functions discussed in ref. ${ }^{43}$ ) are in fact

$$
\tilde{J}_{\mu}(\eta)=\frac{1}{\Gamma(\mu+1)} \int_{0}^{\infty} \frac{x^{\mu}}{1+\exp (x-\eta)} \mathrm{d} x
$$

which, for any real non-negative integer $\mu$, differ from $J_{\mu}(\eta)$, eq. (A.1), only by a factor. The real functions $\tilde{J}_{\mu}(\eta)$ (for real $\eta$ ) are analytic for arbitrary values of $\mu$. (For negative integer $\mu$, the pole occurring in the integral of eq. (A.2) cancels that of the gamma function.) They obey the differential recurrence relation

$$
\frac{\mathrm{d}}{\mathrm{d} \eta} \tilde{J}_{\mu}(\eta)=\tilde{J}_{\mu-1}(\eta)
$$

For $\mu=0,-1,-2, \ldots$ they are elementary functions. In particular one finds

$$
\left.\begin{array}{c}
\tilde{J}_{0}(\eta)=J_{0}(\eta)=\ln \left(1+\mathrm{e}^{\eta}\right), \\
\tilde{J}_{-1}(\eta)=\left(1+\mathrm{e}^{-\eta}\right)^{-1}, \text { etc. }
\end{array}\right\}
$$

Since in the present context we are mostly interested in the real functions with non-integer $\mu$, we choose to stay with the convention according to eq. (A.1) and define the $J_{\mu}(\eta)$ for $\mu<-1$ by the derivative relation

$$
J_{\mu-1}(\eta)=\frac{1}{\mu} \frac{\mathrm{d}}{\mathrm{d} \eta} J_{\mu}(\eta) \quad(\mu \neq 0,-\mu \notin \mathbb{N}=\{1,2,3, \ldots\})
$$

which leads to analytic functions for any real non-negative integer $\mu$.

Since the integral in eq. (A.1) does not exist for $\mu<-1$, we had to look for numerically stable ways to calculate the $J_{-3 / 2}(\eta), J_{-5 / 2}(\eta)$, etc. (The functions for $\mu=\frac{3}{2}, \frac{1}{2}$ and $-\frac{1}{2}$ are easily found in standard computer program libraries; we used the CERN library routine "FERDIR".) Numerical differentiation works well to obtain $J_{-3 / 2}(\eta)$ but becomes unstable for the higher derivatives due to the way in which $J_{-1 / 2}(\eta)$ is approximated. For this reason we took from ref. ${ }^{43}$ ) the expansion

$$
\begin{gathered}
J_{\mu}(\eta)=\Gamma(\mu+1) \sum_{n=0}^{N} \frac{\eta^{n}}{n !}\left(1-2^{n-\mu}\right) \zeta(\mu+1-n) \\
+\frac{2 \pi}{\sin (\pi \mu)} \sum_{k=0}^{\infty}\left\{\left(r_{k}^{2}+\eta^{2}\right)^{\mu / 2} \cos \left[\frac{1}{2} \pi \mu+\mu \operatorname{arctg}\left(\eta / r_{k}\right)\right]\right. \\
\left.\quad-r_{k}^{\mu} \sum_{n=0}^{N} \frac{1}{n !} \frac{\Gamma(\mu+1)}{\Gamma(\mu+1-n)}\left(\frac{\eta}{r_{k}}\right)^{n} \cos \left[(n+\mu) \frac{1}{2} \pi\right]\right\} \\
(\mu<N-1 ; N \in \mathbb{N} ;-\mu \notin \mathbb{N}),
\end{gathered}
$$


where

$$
r_{k}=\pi(2 k+1)
$$

Deriving both sides of eq. (A.6) with respect to $\eta$ and using the relation

$$
\begin{aligned}
J_{\mu}(0) & =\Gamma(\mu+1)\left(1-2^{-\mu}\right) \zeta(\mu+1) \\
& =\frac{\pi^{\mu+1}}{\sin \left(\frac{1}{2} \pi \mu\right)} \sum_{k=0}^{\infty}(2 k+1)^{\mu} \quad(\mu<-1 ;-\mu \notin \mathbb{N}),
\end{aligned}
$$

which is found using standard relations for the Riemann zeta function $\zeta(z)$, we obtain the alternative expansion

$$
\begin{gathered}
J_{\mu}(\eta)=\frac{2 \pi}{\sin (\pi \mu)} \sum_{k=0}^{\infty}\left(r_{k}^{2}+\eta^{2}\right)^{\mu / 2} \cos \left[\frac{1}{2} \pi \mu+\mu \operatorname{arctg}\left(\eta / r_{k}\right)\right] \\
(\mu<-1 ;-\mu \notin \mathbb{N}) .
\end{gathered}
$$

Both eqs. (A.9) and (A.6) can be used for the numerical computation of $J_{\mu}(\eta)$ with non-integer $\mu<-1$; the series (A.6) converges faster but requires the knowledge of $J_{\mu}(0)$ which may be obtained by numerical differentiation of $J_{\mu+1}(\eta)$ at $\eta=0$.

For $\mu=-\frac{3}{2}$ the convergence of these series is too slow for practical use; we therefore calculated $J_{-3 / 2}(\eta)$ by numerical differentiation of $J_{-1 / 2}(\eta)$. For $\mu \leqslant-\frac{5}{2}$, convergence to a relative accuracy of $10^{-5}$ could be achieved using less than 550 items (in most cases 10-50) of the above series.

For values $\eta \leqq-1.5$, faster numerical convergence is found with the following well-known expansion ${ }^{19}$ ):

$$
J_{\mu}(\eta)=\Gamma(\mu+1) \sum_{k=1}^{\infty}(-1)^{k-1} \frac{1}{k^{\mu+1}} \mathrm{e}^{k \eta} \quad(\eta<0) .
$$

Another well-known asymptotic expansion for large positive arguments is ${ }^{19}$ )

$$
J_{\mu}(\eta) \underset{\eta \rightarrow 1}{\sim} \frac{\eta^{\mu+1}}{(\mu+1)}\left\{1+\sum_{k=1}^{\infty}\left(2^{2 k}-2\right) \pi^{2 k}\left|B_{2 k}\right|\left(\begin{array}{c}
\mu+1 \\
2 k
\end{array}\right) \eta^{-2 k}\right\},
$$

where $B_{2 k}$ are the Bernoulli numbers. The series (A.11) is, however, semi-convergent and can therefore only be used for a reliable numerical computation for sufficiently large values of $\eta$. For the Fermi integrals $J_{\mu}(\eta)$ with $-\frac{11}{2} \leqslant \mu \leqslant-\frac{5}{2}$ the convergence of the series (A.11) is so fast for $\eta \geq 20$ that it can be limited to 5 to 9 items to obtain a relative accuracy of $10^{-5}$.

\section{Appendix B}

FOURTH-OR DFR CORRFCTIONS TO THF DFNSITY FUNCTTONAIS

In this appendix we sketch the derivation of the fourth-order gradient corrections to the functionals of the free energy and the entropy in the case of a local potential 
$V(r)$. (The extension to the nonlocal case is lengthy but straightforward and follows the same lines as the local case sketched here). The Bloch-density up to fourth order in the Wigner-Kirkwood expansion reads ${ }^{26}$ )

$$
\begin{aligned}
C_{\mathrm{WK}}(\boldsymbol{r}, \beta)=C_{\mathrm{TF}}(\boldsymbol{r}, \beta)\{1 & +\frac{\hbar^{2}}{12 m}\left[\frac{1}{2} \beta^{3}(\nabla V)^{2}-\beta^{2} \Delta V\right] \\
& \left.+\left(\frac{\hbar^{2}}{2 m}\right)^{2} \frac{1}{60}\left[-\beta^{3} \nabla^{4} V+\beta^{4} C_{4}-\beta^{5} d_{4}+\frac{5}{24} \beta^{6}(\nabla V)^{4}\right]\right\},
\end{aligned}
$$

where

$$
\begin{aligned}
& c_{4}(\boldsymbol{r})=\frac{5}{6}(\Delta V)^{2}+\frac{4}{3} \nabla V \cdot \nabla^{3} V+\frac{1}{3} \Delta(\nabla V)^{2}, \\
& d_{4}(\boldsymbol{r})=\frac{5}{6} \Delta V(\nabla V)^{2}+{ }_{2}^{1} \nabla V \cdot \nabla(\nabla V)^{2} .
\end{aligned}
$$

As shown in subsect. 2.2, we obtain by inverse Laplace transformation of eq. (B.1) the density $\rho_{\mathrm{ETF}}(\boldsymbol{r})$ and the free energy density $\mathscr{F}_{\mathrm{ETF}}(\boldsymbol{r})$ :

$$
\begin{aligned}
& \rho_{E T F}(\boldsymbol{r})=\rho_{\mathrm{TF}}\left(\eta_{0}\right)+\rho_{2}\left(\boldsymbol{r}, \eta_{0}\right)+\rho_{4}\left(\boldsymbol{r}, \eta_{0}\right)=: \rho(\boldsymbol{r}), \\
& \tilde{\mathscr{F}}_{\mathrm{ETF}}(\boldsymbol{r})=\tilde{\mathscr{F}}_{\mathrm{TF}}\left(\eta_{0}\right)+\tilde{\mathscr{F}}_{2}\left(\boldsymbol{r}, \eta_{0}\right)+\tilde{\mathscr{F}}_{4}\left(\boldsymbol{r}, \eta_{0}\right),
\end{aligned}
$$

where

$$
\eta_{0}(\boldsymbol{r})=(\lambda-V(\boldsymbol{r})) / T \text {. }
$$

As in subsect. 2.2, we denote $\rho_{\mathrm{ETF}}(\boldsymbol{r})$, eq. (B.3), in short by $\rho$ which will be the variational density in the applications of the functionals $\mathscr{F}_{\mathrm{ETF}}[\rho]$ and $\sigma_{\mathrm{ETF}}[\rho]$ to be derived here. The explicit expressions of the zeroth- (TF) and second-order terms have already been given in subsect. 2.2 ; that of $\rho_{4}\left(r, \eta_{0}\right)$ will not be needed, as we will show, and that of $\tilde{\mathscr{F}}_{4}\left(r, \eta_{0}\right)$ is

$$
\begin{aligned}
\tilde{\mathscr{F}}_{4}\left(\boldsymbol{r}, \eta_{0}\right)=-A_{T}\left(\frac{\hbar^{2}}{2 m}\right)^{2} \frac{1}{240}[ & \frac{\nabla^{4} V}{T^{2}} J_{-3 / 2}\left(\eta_{0}\right)+\frac{3}{2} \frac{c_{4}}{T^{3}} J_{-5 / 2}\left(\eta_{0}\right) \\
& \left.+\frac{15}{4} \frac{d_{4}}{T^{4}} J_{-7 / 2}\left(\eta_{0}\right)+\frac{175}{64} \frac{(\nabla V)^{4}}{T^{5}} J_{-9 / 2}\left(\eta_{0}\right)\right]
\end{aligned}
$$

with

$$
A_{T}=\frac{1}{2 \pi^{2}}\left(\frac{2 m T}{\hbar^{2}}\right)^{3 / 2} .
$$

As in subsect. 2.2 we define $\eta$ as the solution of the equation

$$
\rho(r)=A_{T} J_{1 / 2}(\eta)
$$


for any value of $\boldsymbol{r}$. We now formally expand $\eta$ up to fourth order:

$$
\eta=\eta_{0}+\eta_{2}+\eta_{4}
$$

where $\eta_{2}, \eta_{4}$ are of order $\hbar^{2}$ and $\hbar^{4}$, respectively, relative to $\eta_{0}$. By a formal Taylor expansion of eq. (B.8) around $\eta_{0}$ and comparison of coefficients of equal order in $\hbar$, one obtains the explicit form of $\eta_{2}$ :

$$
\eta_{2}=\frac{2}{A_{T}} \frac{\rho_{2}\left(\boldsymbol{r}, \eta_{0}\right)}{J_{-1 / 2}\left(\eta_{0}\right)}=\frac{\hbar^{2}}{2 m} \frac{1}{12} e_{2} \frac{1}{J_{-1 / 2}\left(\eta_{0}\right)}
$$

with

$$
e_{2}\left(\boldsymbol{r}, \eta_{0}\right)=\frac{3}{4} \frac{(\nabla V)^{2}}{T^{3}} J_{-5 / 2}\left(\eta_{0}\right)+\frac{\Delta V}{T^{2}} J_{-3 / 2}\left(\eta_{0}\right)
$$

the explicit form of $\eta_{4}$ will not be needed, as we shall immediately see.

We now expand $\tilde{\mathscr{F}}_{4}\left(\boldsymbol{r}, \eta_{0}\right)$, eq. (B.6), around $\eta$, using eq. (A.5), and keep consistently all terms up to order $\hbar^{4}$ :

$$
\begin{aligned}
\mathscr{F}_{\mathrm{ETF}}(\boldsymbol{r})= & \tilde{\mathscr{F}}_{\mathrm{TF}}(\eta)-\left(\eta_{2}+\eta_{4}\right) T \rho+\left(\eta_{2}+\eta_{4}\right) A_{T} T J_{1 / 2}(\eta) \\
& -\frac{1}{4} A_{T} T \eta_{2}^{2} J_{-1 / 2}(\eta) \\
& +\tilde{F}_{2}(\boldsymbol{r}, \eta)-\left.\eta_{2} \frac{\partial}{\partial \eta_{0}} \tilde{\mathscr{F}}_{2}\left(\boldsymbol{r}, \eta_{0}\right)\right|_{\eta}+\tilde{\mathscr{F}_{4}}(\boldsymbol{r}, \eta) .
\end{aligned}
$$

The second and third terms cancel due to eq. (B.8). Noting from eqs. (2.43), (2.44) and (3.6) that

$$
\frac{\partial}{\partial \eta_{0}} \tilde{\mathscr{F}_{2}}\left(\boldsymbol{r}, \eta_{0}\right)=-T \rho_{2}\left(\boldsymbol{r}, \eta_{0}\right)
$$

and using the first equality in (B.10), we obtain

$$
\mathscr{F}_{\mathrm{ETF}}(\boldsymbol{r})=\tilde{\mathscr{F}}_{\mathrm{TF}}(\eta)+\tilde{\mathscr{F}}_{2}(\boldsymbol{r}, \eta)+\tilde{\mathscr{F}}_{4}(\boldsymbol{r}, \eta)+\frac{1}{4} A_{T} T \eta_{2}^{2} J_{-1 / 2}(\eta) .
$$

$\mathscr{F}_{\mathrm{ETF}}(\boldsymbol{r})$, eq. (B.13), now only depends on functions of $\eta$ (and therefore of $\rho$ ) and of the potential $V$ and its gradients. In order to get rid of the latter, we build the corresponding combinations of second, third and fourth derivatives of $\rho$, using eqs. (B.3) and (B.8), but keeping consistently only those terms which contribute to $\mathscr{F}_{\text {ETF }}(\boldsymbol{r})$ in second and fourth order in $\hbar$. After some tedious algebra one can express all gradients of $V$ through combinations of gradients of $\rho$, and one finally obtains the functional for the free energy density:

$$
\mathscr{F}_{\mathrm{ETF}}(\boldsymbol{r})=\mathscr{F}_{\mathrm{TETF}}[\rho]=\mathscr{F}_{\mathrm{TF}}(\rho)+\mathscr{F}_{2}[\rho]+\mathscr{F}_{4}[\rho],
$$


where $\mathscr{F}_{\mathrm{TF}}(\rho)$ and $\mathscr{F}_{2}[\rho]$ have been given in subsect. 3.1 and $\mathscr{F}_{4}[\rho]$ bas the form

$$
\begin{aligned}
\mathscr{F}_{4}[\rho]= & \left(\frac{h^{2}}{2 m}\right)^{2} \frac{1}{T} \frac{J_{-1 / 2}(\eta)}{J_{1 / 2}(\eta)} \\
& \times\left[\phi_{1} \nabla^{4} \rho+\phi_{2} \frac{(\Delta \rho)^{2}}{\rho}+\phi_{3} \frac{\nabla \rho \cdot \nabla^{3} \rho}{\rho}+\phi_{4} \frac{\Delta(\nabla \rho)^{2}}{\rho}+\phi_{5} \frac{\nabla \rho \cdot \nabla(\nabla \rho)^{2}}{\rho^{2}}\right. \\
& \left.+\phi_{6} \frac{\Delta \rho(\nabla \rho)^{2}}{\rho^{2}}+\phi_{7} \frac{(\nabla \rho)^{4}}{\rho^{3}}\right] .
\end{aligned}
$$

The $\phi_{i}$ are universal functions of $\eta$ which can be expressed in terms of the following combinations of $J_{\nu}(\eta)$ :

$$
\begin{gathered}
x=J_{1 / 2} J_{-3 / 2} / J_{-1 / 2}^{2}, \quad y=J_{1 / 2}^{2} J_{-5 / 2} / J_{-1 / 2}^{3}, \quad z=J_{1 / 2}^{3} J_{-7 / 2} / J_{-1 / 2}^{4}, \\
w=J_{1 / 2}^{4} J_{-9 / 2} / J_{-1 / 2}^{5}, \quad v=J_{1 / 2}^{5} J_{-11 / 2} / J_{-1 / 2}^{6} .
\end{gathered}
$$

Here and in the following, the argument of $J_{\nu}$ is always understood to be $\eta$. The expressions for the $\phi_{1}$ are

$$
\begin{gathered}
\phi_{1}=-\frac{1}{180} x, \quad \phi_{2}=-\frac{1}{80} x^{2}+\frac{1}{48} y, \quad \phi_{3}=-\frac{1}{40} x^{2}+\frac{1}{20} y, \\
\phi_{4}=-\frac{1}{180} x^{2}-\frac{1}{80} y, \quad \phi_{5}=-\frac{17}{360} x^{3}+\frac{37}{240} x y-\frac{7}{48} z, \\
\phi_{6}=-\frac{3}{40} x^{3}+\frac{7}{30} x y-\frac{5}{24} z, \\
\phi_{7}=-\frac{7}{48} x^{4}+\frac{5}{8} x^{2} y-\frac{87}{320} y^{2}-\frac{11}{16} x z+\frac{35}{64} w .
\end{gathered}
$$

In practice we only need the functional $\mathscr{F}_{4}[\rho]$ integrated over all space. Assuming that all derivatives of $\rho$ vanish at infinity, we can integrate eq. (B.15) by parts to obtain

$$
F_{4}[\rho]=\int \mathscr{F}_{4}[\rho] \mathrm{d}^{3} r=\left(\frac{\hbar^{2}}{2 m}\right)^{2} \frac{1}{T} \int\left[\theta_{1} \frac{(\Delta \rho)^{2}}{\rho}+\theta_{2} \frac{\Delta \rho(\nabla \rho)^{2}}{\rho^{2}}+\theta_{3} \frac{(\nabla \rho)^{4}}{\rho^{3}}\right] \mathrm{d}^{3} r,
$$

so that only first and second derivatives of $\rho$ will be required. The coefficients $\theta_{i}(\eta)$ are

$$
\begin{gathered}
\theta_{1}=\frac{J_{-1 / 2}}{J_{1 / 2}}\left(\frac{1}{144} x^{2}-\frac{1}{80} y\right), \quad \theta_{2}=\frac{J_{-1 / 2}}{J_{1 / 2}}\left(\frac{1}{72} x^{3}-\frac{11}{240} x y+\frac{1}{24} z\right), \\
0_{3}=\frac{J_{-1 / 2}}{J_{1 / 2}}\left(\frac{1}{144} x^{4}-\frac{1}{30} x^{2} y+\frac{1}{64} y^{2}+\frac{1}{24} x z-\frac{7}{192} w\right) .
\end{gathered}
$$

From eq. (B.18) we finally find the functional for the integrated entropy from the 
canonical relation $S=\left.(-\partial / \partial T) F\right|_{\rho}$. Using

$$
\left.\frac{\partial \eta}{\partial T}\right|_{\rho}=-\frac{3}{T} \frac{J_{1 / 2}(\eta)}{J_{-1 / 2}(\eta)},
$$

which together with the relation (A.5) follows directly from eqs. (B.7), (B.8), one arrives at

$$
S_{4}[\rho]=\int \sigma_{4}[\rho] \mathrm{d}^{3} r=\left(\frac{\hbar^{2}}{2 m}\right)^{2} \frac{1}{T^{2}} \int\left[\chi_{1} \frac{(\Delta \rho)^{2}}{\rho}+\chi_{2} \frac{\Delta \rho(\nabla \rho)^{2}}{\rho^{2}}+\chi_{3} \frac{(\nabla \rho)^{4}}{\rho^{3}}\right] \mathrm{d}^{3} r
$$

where the coefficients $\chi_{i}(\eta)$ are given by

$$
\chi_{i}=\theta_{i}+3 \frac{J_{1 / 2}}{J_{-1 / 2}} \frac{\mathrm{d} \theta_{i}}{\mathrm{~d} \eta} \quad(i=1,2,3)
$$

and take the explicit form

$$
\begin{gathered}
\chi_{1}=\frac{J_{-1 / 2}}{J_{1 / 2}}\left(\frac{5}{288} x^{2}-\frac{1}{32} y-\frac{1}{10} x y+\frac{1}{32} x^{3}+\frac{3}{32} z\right) \\
\chi_{2}=\frac{J_{-1 / 2}}{J_{1 / 2}}\left(\frac{1}{18} x^{3}+\frac{5}{48} x^{4}-\frac{11}{60} x y-\frac{37}{80} x^{2} y+\frac{1}{6} z+\frac{17}{32} z x+\frac{33}{160} y^{2}-\frac{7}{16} w\right) \\
\chi_{3}=\frac{J_{-1 / 2}}{J_{1 / 2}}\left(\frac{11}{288} x^{4}+\frac{7}{96} x^{5}-\frac{11}{60} x^{2} y-\frac{17}{40} x^{3} y+\frac{267}{640} x y^{2}+\frac{11}{128} y^{2}\right. \\
\left.\quad+\frac{11}{48} x z+\frac{9}{16} x^{2} z-\frac{27}{64} y z-\frac{77}{384} w-\frac{21}{32} x w+\frac{63}{128} v\right)
\end{gathered}
$$

The functions $\theta_{i}(\eta)$ and $\chi_{i}(\eta)$ can be computed once and for all.

With the asymptotic expansions (A.10) and (A.11) of the $J_{p}(\eta)$, the quantities $x, y, z, w, v$, eq. (B.16) can be shown to become constants in the limits $\eta \ll 0$ and $\eta \gg 1$ and the $\theta_{i}$ and $\chi_{i}$ take simple values which are shown in table 4 . Note in particular that for arbitrary finite values of $\rho, \eta$ goes to $+\infty$ like $1 / T$ in the limit $T \rightarrow 0$ as explained in sect. 3 ; then the integrand of $S_{4}$ everywhere goes to zero linearly with $T$ and $\mathscr{F}_{4}[\rho]$ goes over to the functional $\left(\hbar^{2} / 2 m\right) \tau_{4}[\rho]$ which is well known from the $T=0$ case $^{13,16}$ ).

TABLE 4

Asymptotic values of the various coefficients defined in appendix $B$ for the two limits $\eta \ll 0$ and $\eta \gg 1$

\begin{tabular}{ccccccccccccc}
\hline & $x$ & $y$ & $z$ & $w$ & $v$ & $\theta_{1}$ & $\theta_{2}$ & $\theta_{3}$ & $\psi_{1}$ & $\psi_{2}$ & $\psi_{3}$ & $J_{-1 / 2} / J_{1 / 2}$ \\
\hline$\ll 0$ & -1 & $1 / 3$ & $-1 / 15$ & $1 / 105$ & $-1 / 945$ & $1 / 180$ & $-1 / 360$ & 0 & $1 / 180$ & $-1 / 360$ & 0 & 2 \\
$\gg 1$ & $-1 / 3$ & $-1 / 27$ & $-1 / 135$ & $-1 / 567$ & $-1 / 2187$ & $1 / 270 \eta$ & $-1 / 240 \eta$ & $1 / 810 \eta$ & 0 & 0 & 0 & $3 / \eta$ \\
\hline
\end{tabular}


The coefficients $\zeta(\eta)$ and $\nu(\eta)$ occurring in the second-order functionals - see eqs. (3.11), (3.17) - can be expressed by the quantities $x$ and $y$ in eq. (B.16) as ${ }^{\dagger}$

$$
\zeta=-\frac{1}{12} x, \quad y=\frac{1}{8}\left(x+2 x^{2}-3 y\right) .
$$

\section{References}

1) A.D. Panogotion et al., Phys. Rev. I.ett. 52 (1984) 496; see also P. Siemens, Nature 305 (1983) 410; Nucl. Phys. A428 (1984) $197 \mathrm{c}$

2) J. Bartel and P. Quentin, Phys. Lett. 152B (1985) 29

3) G.E. Brown, H.A. Bethe and G. Baym, Nucl. Phys. A375 (1982) 481; see also J.M. Lattimer, Ann. Rev. Nucl. Part. Sci. 31 (1981) 337

4) See e.g. H.N.V. Temperley, Proc. Phys. Soc. 59 (1947) 199

5) M. Brack and P. Quentin, Phys. Lett. 52B (1974) 159; Phy. Scripta A10 (1974) 163

6) U. Mosel, P. Zint and K.H. Passler, Nucl. Phys. A236 (1974) 252

7) P. Bonche and D. Vautherin, Nucl. Phys. A372 (1981) 496; Astron, Astrophys. 112 (1982) 168

8) R. Wolf, Dissertation, München (1984)

9) P. Bonche, S. Levit and D. Vautherin, Nucl. Phys. A427 (1984) 278

10) M. Brack, C. Guet and H.-B. Håkansson, Phys. Reports 123 (1985) 275

11) V.M. Strutinsky, Nucl. Phys. A95 (1967) 420; A122 (1968) 1

12) M. Brack and P. Quentin, Nucl. Phys. A361 (1981) 35

13) D.A. Kirzhnits, Field theoretical methods in many body systems (Pergamon, Oxford, 1967);

B. Grammaticos and A. Voros, Ann. of Phys. 123 (1979) 359; 129 (1980) 153

14) L.H. Thomas, Proc. Camb. Phil. Soc. 23 (1926) 542;

E. Fermi, Acc. Lencei 6 (1927) 602;

C.F. v. Weizsäcker, Z. Phys. 96 (1935) 431;

H.A. Bethe and F. Bacher, Rev. Mod. Phys. 8 (1936) 82

15) P. Hohenberg and W. Kohn, Phys. Rev. 136B (1964) 864;

see also M. Levy and J. Perdew, in Density functional methods in physics, ed. R. Dreizler and J. da Providencia (Plenum, New York, 1985) p. 11

16) M. Brack, B.K. Jennings and Y.H. Chu, Phys. Lett. 65B (1976)1;

C. Guet and M. Brack, Z. Phys. A297 (1980) 247

17) C. Guet, H.-B. Håkansson and M. Brack. Phys. Lett. 97B (1980) 7

18) N.D. Mcrmin, Phys. Rcv. 137 (1965) ^ 1441

19) E.C. Stoner, Phil. Mag. 28 (1939) 257

20) W.A. Küpper, G. Wegmann and E. Hilf, Ann. of Phys. 88 (1974) 454

M. Barranco and I.R. Buchler, Phys. Rev. C22 (1980) 1729;

D.Q. Lamb et al., Nucl. Phys. A360 (1981) 459

21) W. Stocker and J. Burzlaff, Nucl. Phys. A202 (1973) 265;

D.G. Ravenhall, C.J. Pethick and J.M. Lattimer, Nucl. Phys. A405 (1983) 571

22) F. Perrot, Phys. Rev. A20 (1979) 586

23) M. Brack, J. de Phys. C6 (1984) 15

24) M. Brack, Phys. Rev. Lett. 53 (1984) 119; 54 (1985) 851

25) E.P. Wigner, Phys. Rev. 40 (1932) 749;

J.G. Kirkwood, Phys. Rev. 44 (1933) 31;

G.E. Uhlenbeck and E. Beth, Physica 3 (1936) 729

26) R.K. Bhaduri and C.K. Ross, Phys. Rev. Lett. 27 (1971) 606;

B.K. Jennings, Nucl Phys. A207 (1973) 538;

B.K. Jennings, R.K. Bhaduri and M. Brack, Nucl. Phys. A253 (1975) 29, and references quoted therein;

B.K. Jennings, Ph.D. thes1s, McMaster (1976), unpublished

$\dagger$ The overall sign of $\nu$ given in refs. ${ }^{23,24}$ ) is wrong. 
27) N.H. March, W.H. Young and S. Sampanthar, The many-body problem in quantum mechanics (Cambridge Univ. Press, Cambridge, 1967)

28) D. Vautherin and D.M. Brink, Phys. Rev. C5 (1972) 626

29) Y.H. Chu, Ph.D. thesis, Stony Brook (1977), unpublished

30) M. Brack and H.C. Pauli, Nucl. Phys. A207 (1973) 401

31) M.J. Giannoni and P. Quentin, Phys. Rev. C21 (1980) 2076

32) J.W. Negele and D. Vautherin, Phys. Rev. C5 (1972) 1472

33) W.D. Myers and W.J. Swiatecki, Nucl. Phys. 81 (1966) 1; Ann. of Phys. 55 (1969) 395; 84 (1974) 186

34) V. Pereyra, in Codes for boundary value problems in ordinary differential equations, eds. B. Childs et al., Lecture Notes in Computer Science, 76 (1979)

35) J. Bartel, M. Brack, C. Guet and H.-B. Håkansson, Phys. Lett. 139B (1984) 1

36) J. Bartel et al., Nucl. Phys. A386 (1982) 79

37) M. Brack et al., Rev. Mod. Phys. 44 (1972) 320

38) H. Beiner et al., Nucl. Phys. A238 (1975) 29

39) H.S. Köhler, Nucl. Phys. A258 (1976) 301

40) H. Krivine, J. Treiner and O. Bohigas, Nucl. Phys. A336 (1980) 155

41) F. Tondeur, Phys. Lett. 123B (1983) 139

42) X. Viñas et al., J. de Phys. C6 (1984) 103

43) F.J. Fernández Velicia, Phys. Rev. A30 (1984) 1194

44) M. Barranco and J. Treiner, Nucl. Phys. A351 (1981) 269

45) J.M. Pearson, M. Farine and J. Côté, Phys. Rev. C26 (1982), and references therein

46) F. Tondeur et al., Nucl. Phys. A420 (1984) 297 